\title{
Design of cold-formed stainless steel lipped channel- sections with web openings subjected to web crippling under End-One-Flange loading condition
}

\author{
Amir M. Yousefi ${ }^{1}$, James B.P. Lim¹, Asraf Uzzaman², Ying Lian³, \\ G. Charles Clifton ${ }^{1}$, Ben Young ${ }^{4}$ \\ ${ }^{1}$ Department of Civil and Environmental Engineering, The University of Auckland, \\ New Zealand \\ ${ }^{2}$ Department of Mechanical and Aerospace Engineering, The University of Strathclyde, \\ 75 Montrose Street, Glasgow G1 1XJ, UK \\ ${ }^{3}$ SPACE, David Keir Building, Queen's University, Belfast, BT9 5AG, UK \\ ${ }^{4}$ Department of Civil Engineering, The University of Hong Kong, Pokfulam Road, \\ Hong Kong
}

(Received: $\quad$; Revised: $\quad$; Accepted:

\begin{abstract}
This paper presents a numerical investigation on the web crippling strength of cold-formed stainless steel lipped channel-sections with circular web openings under end-one-flange (EOF) loading condition. In order to take into account the influence of the circular web openings, a parametric study involving 1,992 finite element analyses was performed, covering duplex EN1.4462, austenitic EN1.4404 and ferretic EN1.4003 stainless steel grades; from the results of the parametric study, strength reduction factor equations are proposed. The web crippling strengths predicted by the reduction factor equations are first compared to the strengths calculated using the equations recently proposed for cold-formed carbon steel lipped channel-sections. It is demonstrated that the strength reduction factor equations proposed for cold-formed carbon steel are unconservative for the stainless steel grades by up to $7 \%$. Unified strength reduction factor equations are then proposed that can be applied to all three stainless steel grades.
\end{abstract}

Key words: Cold-formed stainless steel, Lipped channel-section, Web crippling, Finite element analysis, Strength reduction factor. 


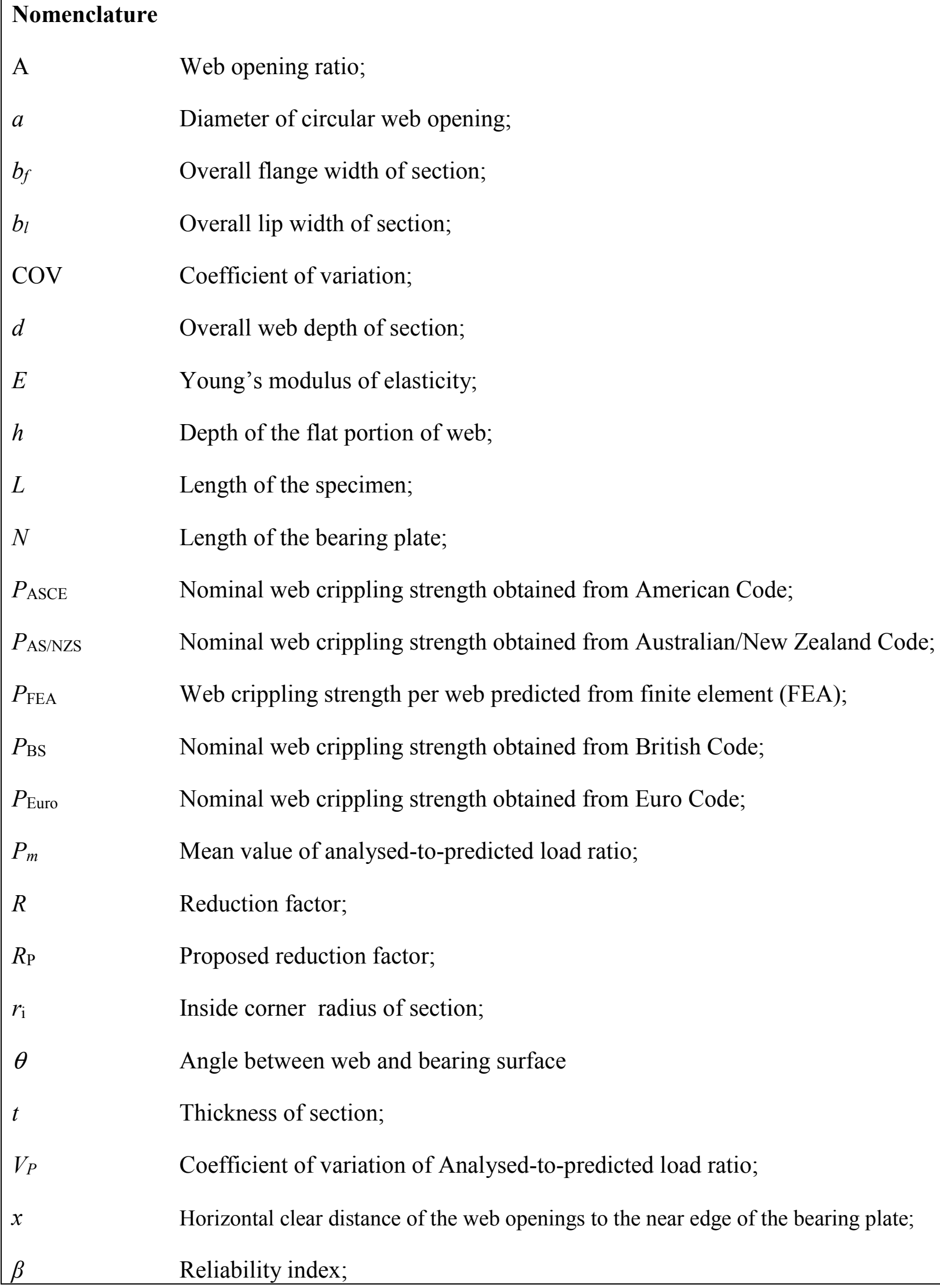




\section{Introduction}

Cold-formed stainless steel sections increasingly are been used in the construction industry, for both architectural as well as structural applications (Nethercot et. al. 2011, Theofanous and Gardner 2011, Kiymaz and Seckin 2014) and the use of web openings in such sections is becoming increasingly popular (Lawson et. al. 2015) (see Figure 1). Such openings, however, result in the sections being more susceptible to web crippling as a form of localized buckling, especially under concentrated loads applied to the bearing flange in the vicinity of the openings.

The authors have recently proposed strength reduction factor equations for the web crippling strength of cold-formed stainless steel lipped channel-sections with circular web openings under the interior-one-flange (IOF) loading condition (Yousefi et al. 2016). The equations covered three stainless steel grades: duplex grade EN 1.4462; austenitic grade EN 1.4404 and ferritic grade EN 1.4003. Other than Yousefi et al. (2016), no previous research has considered the web crippling strength of cold-formed stainless steel lipped channelsections with circular web openings under either of the one or two-flange loading conditions. The work extended that of Lian et al. $(2016 \mathrm{a}, \mathrm{b})$ considering cold-formed stainless steel instead of cold-formed carbon steel. Conducting a parametric study of 2,218 cold-formed stainless steel lipped channel-sections with various dimensions and thicknesses, the strength reduction factor equations proposed by Lian et al. $(2016 \mathrm{a}, \mathrm{b})$ were shown to be conservative by $2 \%$ for the duplex grade and around $9 \%$ for the austenitic and ferritic grades.

For cold-formed carbon steel with circular web openings, Lian et al. (2016c,d) have considered the end-one-flange (EOF) loading condition. The work of Lian et al. $(2016 \mathrm{c}, \mathrm{d})$ was a continuation of the work of Uzzaman et al. (2012a,b,c, 2013), who considered the two-flange loading condition. For cold-formed stainless steel lipped channel-sections without openings, only Kroyink et al. (1996) has considered the web crippling strength. 
Zhou and Young $(2006,2007,2008,2013)$ have considered the web crippling strength of cold-formed stainless steel tubular sections; Keerthan and Mahendran (2012) and Keerthan et. al. (2014) considered the web crippling strength of hollow flange channel beams. Research by Lawson et al. (2015), while concerned with circular web openings, focussed on the bending strength of the sections and not on the web crippling strength under concentrated loads.

This paper considers the case of the web crippling strength of cold-formed stainless steel lipped channel-sections under the end-one-flange (EOF) loading condition (see Figures 2 and 3) and applicability of the proposed equations by Lian et al. (2016c,d) to three stainless steel grades, namely the duplex grade EN 1.4462; austenitic grade 1.4404 and ferritic grade 1.4003. Typical stress-strain curves for the three grades were taken from Chen and Young (2006) and Arrayago et. al. (2015).

\section{Experimental investigation and finite element modelling}

For cold-formed carbon steel, Lian et al. $(2016 \mathrm{c}, \mathrm{d})$ recently conducted 74 end-oneflange (EOF) laboratory tests on lipped channel-sections with circular web openings subjected to web crippling (see Figure 3). Figure 4 shows the definition of the symbols used to describe the dimensions of the cold-formed carbon steel lipped channel-sections considered in the test programme. The laboratory tests were used to validate a non-linear geometry elasto-plastic finite element model in ABAQUS (2014), which was then used for a parametric study, from which design recommendations are proposed in the form of strength reduction factor equations, relating the loss of strength due to the holes to the strength of the web without openings. The size of the circular web openings was varied in order to investigate the effect of the web opening size on the web crippling strength. Full details of 
both the laboratory tests and finite element models (see Figure 5) can be found in Lian et al. $(2016 c, d)$.

The models have been labeled such the nominal dimension of the model and the length of the bearing plate as well as the ratio of the diameter of the circular web openings to the depth of the flat portion of the webs $(a / h)$ can be determined from the labeling system. As an example, the label "142-N100-A0.2-FR" means the following. The first notation is the nominal depth of the models in millimeters. The notation "N100" indicates the length of bearing in millimeters (i.e. $100 \mathrm{~mm}$ ). The notation "A0.2" indicates the ratio of the diameter of the openings to the depth of the flat portion of the webs $(a / h)$ and are one of $0.2,0.4,0.6$ and 0.8 (i.e. $A 0.2$ means $a / h=0.2 ; A 0.4$ means $a / h=0.4$ etc). Plain lipped channel-sections (i.e. without circular web openings) are denoted by " $A 0$ ". The flange unfastened and fastened cases are identified as "FR" and " $F X "$, respectively.

Figure 6 compares the experimental and numerical load-displacement curves for a cold-formed carbon steel lipped channel-section, $142 \times 60 \times 13-\mathrm{t} 1.3-\mathrm{N} 100-\mathrm{FR}$, covering the cases both with and without the circular web openings. As can be seen, there is good agreement between the failure loads of the tested specimens and the finite element results. While there is a difference in the post-ultimate range of the load-displacement curves, where larger displacements were found in the tests compared with the numerical predictions, this difference can be attributed to the fact that in the finite element model linear cartesian connector elements were used to simulate bolt-hole elongation instead of physically modelling the bolts. The stiffness of the connector elements was $10 \mathrm{kN} / \mathrm{mm}$, which Lim and Nethercot $(2004 a, b)$ suggest would be suitable. In addition, further displacement in the postultimate range of the load-displacement curves can be attributed to slip between the channel sections and the test rig, which was observed in the laboratory tests. 
For cold-formed stainless steel lipped channel-sections, the numerical failure loads with and without circular web openings were then determined for the three stainless steel grades: duplex grade EN 1.4462; austenitic grade 1.4404 and ferritic grade 1.4003 (see Table 1). These results were compared with the failure loads calculated in accordance with ASCE 8-02 (2002), BS 5950-5 (1998) and Eurocode-3 (2006) (see Table 2). The failure loads predicted from the finite element analyses are generally similar to the standard codified failure loads of the sections.

\section{Parametric study for stainless steel grades}

In this study, in order to investigate the effect of circular web openings on the web crippling strength of cold-formed stainless steel lipped channel-sections, a total of 1,992 finite element models of lipped channel-sections with various dimensions and thicknesses were considered for the three stainless steel grades: duplex EN1.4462, austenitic EN1.4404 and ferritic EN1.4003. The web crippling strength predicted was influenced primarily by the ratio of the circular web opening depth to the flat portion of the web, the ratio of the bearing length to the flat portion of the web and the location of the web opening as defined by the distance of the web opening from the edge of the bearing divided by the flat portion of the web (Lian et al. (2016a,b) and Uzzaman et al. (2012a,b,c, 2013)). In order to find the effect of $\mathrm{a} / \mathrm{h}, \mathrm{N} / \mathrm{h}$ and $\mathrm{x} / \mathrm{h}$ on the web crippling strength of channel sections with circular web openings, parametric studies were carried out considering the circular web openings, different bearing plate lengths, the cross-section sizes and location of the circular web openings. The cases of both flanges fastened and flanges unfastened to the bearing plates were considered.

The specimens consisted of three different section sizes, having thicknesses $(\mathrm{t})$ ranging from $1.23 \mathrm{~mm}$ to $6.0 \mathrm{~mm}$ and web slenderness $(\mathrm{h} / \mathrm{t})$ values ranging from 111.7 to 157.8 . The 
ratios of the diameter of the circular web openings (a) to the depth of the flat portion of the webs (h) were $0.2,0.4,0.6$ and 0.8 . The ratios of the distance of the web openings ( $\mathrm{x}$ ) to the depth of the flat portion of the web (h) were $0.2,0.4$ and 0.6 . Bearing plates of lengths $(\mathrm{N})$ equal to $100 \mathrm{~mm}, 120 \mathrm{~mm}$ and $150 \mathrm{~mm}$ are considered. For each series of specimens, the web crippling strengths of the sections without the web openings were obtained. Thus, the ratio of the web crippling strengths for sections with web openings divided by the sections without web openings, which is the strength reduction factor (R), was used to quantify the degrading influence of the web openings on the web crippling strengths.

A total of 1,992 specimens was analysed in the parametric study investigating the effect of the ratios of $a / h, N / h$ and $x / h$. The web crippling strengths ( $\left.\mathrm{P}_{\mathrm{FEA}}\right)$ per web predicted from the FEA are summarised in Tables 3 to 5 for the duplex grade EN 1.4462, austenitic grade 1.4404 and ferritic grade 1.4003. As can be seen from Tables 3 to 5, the failure load of the cold-formed stainless steel sections reduces as web openings are present and continues to reduce with increase in the size of web openings. The results demonstrate that the failure load obtained from the cold-formed stainless steel sections with the case of flanges fastened to bearing plate is in average 30\% higher than the case of flanges unfastened to bearing plates for the sections with and without web openings. In addition, for the case of web openings with a horizontal clear distance to the near edge of the bearing plate, the web crippling strength of the sections is higher than the case of web openings located centred above the bearing plates. Moreover, for the same section with different span and bearing plate, the failure loads were found to be different among the results. Based on the results, it was found that the failure load increases as the length of the bearing plates increases and as the length of the sections increases. The effect of the ratios of $\mathrm{a} / \mathrm{h}$ and $\mathrm{x} / \mathrm{h}$ on the reduction factor of the web crippling strength is shown in Figure 7 for the C142 specimen. 
Figure 7(a) shows the ratio of the circular web opening depth to the flat portion of the web $(a / h)$ versus the strength reduction factor, for the three stainless steel grades. As can be seen, the reduction in strength increases as the parameter $a / h$ increases for all three stainless steels, in particular for the ferritic grade with lower thickness $(1.3 \mathrm{~mm})$. The reduction in strength of the ferritic grade $6 \mathrm{~mm}$ thick section is smallest and the reduction in strength increases as the section becomes thinner. It can be seen that when the $a / h$ ratio increases from 0.2 to 0.6 , the reduction in strength for the ferritic grade increases by $29 \%$. It also can be seen that for sections of $4 \mathrm{~mm}$ thickness, due to different mechanical properties and more rounded stress-strain curves of austenitic and ferritic stainless steel grades, compared to the duplex grade, that there was a small reduction in strength for those two grades. From Figure 7(b) it can be seen that the reduction in strength is sensitive to the horizontal distance of the web openings to the bearing plate. As the ratio of $x / h$ decreases from 0.6 to 0.2 , the strength reduction factor decreases by $7 \%$. Also, it can again be seen that the reduction in strength is less for the austenitic grade compared to that of the other two stainless steel grades.

\section{Reduction factor comparison with Lian et al. (2016c,d)}

For ease of reference, the reduction factor equations proposed by Lian et al. (2016c,d) are summarised below:

For centered web opening:

Free case $\quad R_{P}=0.96-0.34\left(\frac{a}{h}\right)+0.09\left(\frac{N}{h}\right)$

Fixed case $\quad R_{P}=0.93-0.41\left(\frac{a}{h}\right)+0.16\left(\frac{N}{h}\right)$

For offset web opening:

Free case $\quad R_{P}=0.97-0.26\left(\frac{a}{h}\right)+0.14\left(\frac{x}{h}\right)$

Fixed case: $R_{P}=0.97-0.14\left(\frac{a}{h}\right)+0.07\left(\frac{x}{h}\right)$ 
where the limits for the reduction factor in Eqns. 1 to 4 are $h / t \leq 157.8, N / t \leq 120.97$, $N / h \leq 1.15, a / h \leq 0.8$, and $\theta=90^{\circ}$.

In order to evaluate the applicability of the proposed equations to cold-formed stainless steel grades, an extensive statistical analysis has been performed on all four proposed equations. Table 6 compares the reduction factors determined from the finite element models to Eqns. 1 to 4 for cases of centred and offset web opening where the flange is unfastened to the bearing plate.

As can be seen from Table 6, the four equations proposed by Lian et al. (2016c,d) for carbon steel are unconservative for the three stainless steel grades, especially for sections with centred web openings. Examining the strength reduction factor ratios obtained from the finite element analyses, with the exception of the offset web opening fixed case which has a mean reduction factor ratio of 1.00 and coefficient of variation $(\mathrm{COV})$ of 0.01 , the other reduction factors from Lian et al. (2016c,d) are unconservative for the stainless steel grades, especially for the ferritic and austenitic stainless steel grades. For example, for the centred web opening case for ferritic grade, the mean value of the web crippling reduction factor ratio is 0.93 and 0.98 for the cases of flange unfastened and fastened to the bearing plate, respectively; the corresponding values of $\mathrm{COV}$ are 0.10 and 0.06 , respectively. In the next section, new equations are proposed for each of the three stainless steel grades.

\section{Proposed strength reduction factors}

Tables 3 to 5 show the dimensions considered and web crippling strengths of the duplex, austenitic and ferritic stainless steel sections predicted from the finite element analysis. Using bivariate linear regression analysis, two unified strength reduction factor equations $\left(R_{\mathrm{p}}\right)$ for three stainless steel grades with web openings are proposed. The equations are as follows: 
Centred web opening $\quad R_{p}=\alpha-\gamma\left(\frac{a}{h}\right)-\lambda\left(\frac{N}{h}\right) \leq 1$

Offset web opening $\quad R_{p}=\rho+\mu\left(\frac{a}{h}\right)+\zeta\left(\frac{x}{h}\right) \leq 1$

The limits for the reduction factor Eqns. 5 and 6 remain $h / t \leq 157.8, N / t \leq 120.97$, $N / h \leq 1.15, a / h \leq 0.8$, and $\theta=90^{\circ}$. The coefficients $\alpha, \gamma, \lambda, \rho, \mu$ and $\zeta$ of the equations are calibrated with the stainless steel analysis results, and the coefficients are presented in Table 7.

\section{Comparison of numerical results with proposed reduction factors}

For the three stainless steels grades, the values of the strength reduction factor $(R)$ obtained from the numerical results are compared with the values of the proposed strength reduction factor $\left(R_{p}\right)$ calculated using Eqns. 5 and 6. The results for C142 are shown in Figure 8 . In order to evaluate the accuracy of proposed equations, extensive statistical reliability analyses are performed. The results are summarized in Table 8 . It should be noted, in calculating the reliability index, the resistance factor of $\phi=0.85$ was used, corresponding to the reliability index $\beta$ from the NAS specification. The load combination of $1.2 \mathrm{DL}+$ 1.6LL as specified in the NAS specification was used in the reliability analysis, where DL is the dead load and LL is the live load. In this study, $M_{m}=1.10$ and $V_{M}=0.10$, which are the mean and coefficients of variation for the material properties factors, $F_{m}=1.00$ and $V_{F}=$ 0.05 , which are the mean and coefficients of variation for the fabrication factors, and $V_{q}=$ 0.21 , which is the coefficient of variation of load effect were used. According to the NAS specification, design rules are reliable if the reliability index is more than 2.5 . As can be seen in Table 8 , the proposed reduction factors are a good match with the numerical results for the both cases of flanges unfastened and flanges fastened to the bearing plates and particularly for the duplex stainless steel grade. 
For example, for the centred circular web opening, the mean value of the web crippling reduction factor ratios are 0.99 and 1.00 for the cases of flange unfastened and flange fastened to the bearing plate, respectively. The corresponding values of COV are 0.09 and 0.08 , respectively. Similarly, the reliability index values $(\beta)$ are 2.62 and 2.69, respectively. For the offset circular web opening, the mean value of the web crippling reduction factor ratios are 1.04 and 1.04 for the cases of flange unfastened and flange fastened to the bearing plate, respectively. The corresponding values of COV are 0.04 and 0.05 , respectively. Similarly, the reliability index values $(\beta)$ are 2.97 and 2.95 , respectively. Therefore, the proposed strength reduction factor equations are able to reliably predict the influence of the circular web openings on the web crippling strengths of cold-formed stainless steel lipped channel-sections under the end-one-flange (EOF) loading condition.

\section{Conclusions}

In this paper, an investigation into the effect of circular web openings on the web crippling strength of cold-formed stainless steel lipped channel-sections under the end-oneflange (EOF) loading condition has been conducted. For this purpose, a parametric study of 1,992 lipped channel-sections of various dimensions and thicknesses were considered for three stainless steel grades: duplex EN1.4462, austenitic EN1.4404 and ferretic EN1.4003. Cases with and without circular web openings were considered with web openings located centred above and offset to the bearing plates.

Strength reduction factor equations have been determined. When the strength reduction factors were compared against those recently proposed for cold-formed carbon steel by Lian et al. $(2016 \mathrm{c}, \mathrm{d})$, it was observed that the cold-formed carbon steel strength reduction factor equations were unconservative for the stainless steel grades by up to $7 \%$. 
Based on finite element results, new unified web crippling strength reduction factor equations are proposed, considering different web opening diameters and location in the web for both cases of flanges unfastened and flanges fastened to the bearing plates. Reliability analysis was performed in order to evaluate the reliability of the proposed strength reduction factor equations. It was demonstrated that the proposed strength reduction factor equations are generally conservative and agree well with the analysis results. The proposed new unified strength reduction factor equations are capable of producing reliable and safe design values when calibrated according to the NAS specification for resistance factor of 0.85 $(\varphi=0.85)$ for the end-one-flange (EOF) loading condition.

\section{Acknowledgements}

The University of Auckland Doctoral Scholarship is greatly acknowledged and the authors declare that they have no conflict of interest. 


\section{References}

ABAQUS. (2014). Analysis User's Manual-Version 6.14-2 ABAQUS Inc., USA.

Arrayago, I., Real, E. and Gardner, L. (2015). "Description of stress-strain curves for stainless steel alloys", Materials \& Design, Vol. 87, pp. 540-552.

ASCE 8-02 (2002). Specification for the Design of Cold-Formed Stainless Steel Structural Members: SEI/ASCE 8-02, Reston, VA.

BS 5950-5 (1998), Structural use of steelwork in buildings, Part 5 Code of practice for the design of cold-formed sections. British Standards Institution, London.

Chen, J., and Young, B. (2006). "Stress-strain curves for stainless steel at elevated temperatures", Engineering Structures, Vol. 28, No. 2, pp. 229-239.

Eurocode-3 (2006), Design of steel structures: Part 1.3: General rules — Supplementary rules for cold-formed thin gauge members and sheeting, in: ENV 1993-1-3, European Committee for Standardization, Brussels, Belgium.

Keerthan, P., and Mahendran, M. (2012). "Shear behaviour and strength of LiteSteel beams with web openings", Advances in Structural Engineering, Vol. 15, No. 2, pp. 171184. 
Keerthan, P., Mahendran, M. and Steau, E. (2014). "Experimental study of web crippling behaviour of hollow flange channel beams under two flange load cases", ThinWalled Structures, Vol. 85, pp. 207-219.

Kiymaz, G. and Seckin, E. (2014). "Behavior and design of stainless steel tubular member welded end connections", Steel and Composite Structures, Vol 17, No. 3, pp. 253269.

Korvink, S.A., van den Berg, G.J. and van der Merwe, P. (1995). "Web crippling of stainless steel cold-formed beams", Journal of Constructional Steel Research, Vol. 34, No. 23, pp. 225-248.

Lawson, R. M., Basta, A. and Uzzaman, A. (2015). "Design of stainless steel sections with circular openings in shear", Journal of Constructional Steel Research, Vol 112, pp. $228-241$.

Lian, Y., Uzzaman A., Lim, J.B.P., Abdelal, G., Nash, D. and Young, B. (2016a). "Effect of web holes on web crippling strength of cold-formed steel channel sections under Interior-One-Flange loading condition-Part I: tests and finite element analysis", Proceeding of The $8^{\text {th }}$ International Conference on Steel and Aluminium Structures, Hong Kong.

Lian, Y., Uzzaman, A., Lim, J.B.P., Abdelal, G., Nash, D. and Young, B. (2016b). "Parametric studies and design recommendations of Cold-Formed steel sections with web openings subjected to web crippling", Proceeding of The $8^{\text {th }}$ International Conference on Steel and Aluminium Structures, Hong Kong.

Lian, Y., Uzzaman A., Lim, J.B.P., Abdelal, G., Nash, D. and Young, B. (2016c). " Effect of Web Holes on Web Crippling Strength of Cold-Formed Steel Channel Sections under End-One-Flange Loading Condition-Part I: Tests and Finite Element Analysis", Thin-Walled Structures.

Lian, Y., Uzzaman, A., Lim, J.B.P., Abdelal, G., Nash, D. and Young, B. (2016d). "Effect of Web Holes on Web Crippling Strength of Cold-Formed Steel Channel Sections under End-One-Flange Loading Condition -Part II: Parametric Study and Proposed Design Equations, Thin-Walled Structures.

Lim, J.B.P. and Nethercot, D.A. (2004a). "Finite element idealisation of a cold-formed steel portal frame", Journal of Structural Engineering, 130:1, pp.78-94. 
Lim, J.B.P. and Nethercot, D.A. (2004b). "Stiffness prediction for bolted momentconnections between cold-formed steel members", Journal of Constructional Steel Research, 60:1, pp.85-107.

NAS (2007). North American Specification for the Design of Cold-Formed Steel Structural Members: American Iron and Steel Institute, AISI S100-2007, AISI Standard.

Nethercot, D. A., Salih, E. L. and Gardner, L. (2011). "Behaviour and design of stainless steel bolted connections", Advances in Structural Engineering, Vol. 14, No. 4, pp. 647-658.

Theofanous, M., and Gardner L. (2012). "Effect of element interaction and material nonlinearity on the ultimate capacity of stainless steel cross-sections", Steel and Composite Structures, Vol. 12, No. 1, pp. 73-92.

Uzzaman, A., Lim, J. B.P., Nash, D, Rhodes J., and Young, B. (2012a). "Web crippling behaviour of cold-formed steel channel sections with offset web holes subjected to end-two-flange loading", Thin-Walled Structures, Vol. 50, pp. 76-86.

Uzzaman, A., Lim, J. B.P., Nash, D, Rhodes J., and Young, B. (2012b). "Cold-formed steel sections with web openings subjected to web crippling under two-flange loading conditions-part I: Tests and finite element analysis", Thin-Walled Structures, Vol. 56 , pp. 38-48.

Uzzaman, A., Lim, J. B.P., Nash, D, Rhodes J., and Young, B. (2012c). "Cold-formed steel sections with web openings subjected to web crippling under two-flange loading conditions-part II: Parametric study and proposed design equations", Thin-Walled Structures, Vol. 56, pp. 79-87.

Uzzaman, A., Lim, J. B.P., Nash, D, Rhodes J., and Young, B. (2013). "Effect of offset web holes on web crippling strength of cold-formed steel channel sections under end-twoflange loading condition", Thin-Walled Structures, Vol. 65, pp. 34-48.

Yousefi, A. M., Lim, J.B.P., Uzzaman, A., Lian, Y., Clifton, G.C. and Young, B. (2016). "Web crippling strength of cold-formed stainless steel lipped channel-sections with web openings subjected to Interior-One-Flange loading condition", Steel and Composite Structures, Vol. 21, No. 3, pp. 629-659.

Zhou, F. and Young, B. (2006). "Yield line mechanism analysis on web crippling of coldformed stainless steel tubular sections under two-flange loading", Engineering Structures, Vol. 28, No. 6, pp. 880-892. 
Zhou, F. and Young, B. (2007). "Cold-formed high-strength stainless steel tubular sections subjected to web crippling", Journal of structural engineering, Vol. 133, No. 3, pp. 368-377.

Zhou, F. and Young, B. (2008). "Web crippling of cold-formed stainless steel tubular sections", Advances in Structural Engineering, Vol. 11, No. 6, pp. 679-691.

Zhou, F. and Young, B. (2013). "Web crippling behaviour of cold-formed duplex stainless steel tubular sections at elevated temperatures", Engineering Structures, Vol. 57, pp.51-62.

\section{List of tables}

Table 1: Dimensions and web crippling strengths predicted from finite element analysis of cold-formed stainless steel lipped channel sections

a: For the case of flange unfastened to the bearing plate

b: For the case of flange fastened to the bearing plate

Table 2: Comparison of numerical results with design strength for the case of flange fastened to the bearing plate without circular web opening

Table 3: Web crippling strengths of duplex stainless steel sections predicted from finite element analysis

a: $a / h$ for centred circular web opening case 
b: $a / h$ for offset circular web opening case

c: $x / h$ for offset circular web opening case

Table 4: Web crippling strengths of austenitic stainless steel sections predicted from finite element analysis

a: $a / h$ for centred circular web opening case

b: $a / h$ for offset circular web opening case

c: $x / h$ for offset circular web opening case

Table 5: Web crippling strengths of ferritic stainless steel sections predicted from finite element analysis

a: $a / h$ for centred circular web opening case

b: $a / h$ for offset circular web opening case

c: $x / h$ for offset circular web opening case

Table 6: Comparison of web crippling strength reduction factor for cold-formed stainless steel lipped channel sections with reduction factor equations proposed by Lian et al. $(2016 \mathrm{c}, \mathrm{d})$

a: Flange unfastened to the bearing plate

b: Flange fastened to the bearing plate

Table 7: Coefficients of the proposed strength reduction factor equations

Table 8: Statistical analysis of strength reduction factor

a: Duplex stainless steel grade

b: Austenitic stainless steel grade

c: Ferritic stainless steel grade 
Table 1: Dimensions and web crippling strengths predicted from finite element analysis of cold-formed stainless steel lipped channel-sections

a: For the case of flange unfastened to the bearing plate

\begin{tabular}{|c|c|c|c|c|c|c|c|c|c|c|c|c|c|c|c|}
\hline \multirow[t]{2}{*}{ Specimen } & Web & Flange & Lip & Thickness & Length & Web & \multicolumn{3}{|c|}{ Duplex } & \multicolumn{3}{|c|}{ Ferritic } & \multicolumn{3}{|c|}{ Austenitic } \\
\hline & $\begin{array}{c}d \\
(\mathrm{~mm})\end{array}$ & $\begin{array}{c}b_{f} \\
(\mathrm{~mm})\end{array}$ & $\begin{array}{c}b_{l} \\
(\mathrm{~mm})\end{array}$ & $\begin{array}{c}t \\
(\mathrm{~mm})\end{array}$ & $\begin{array}{c}L \\
(\mathrm{~mm})\end{array}$ & $\begin{array}{c}a \\
(\mathrm{~mm})\end{array}$ & $\begin{array}{c}P(A 0) \\
(k N)\end{array}$ & $\begin{array}{l}\text { P(Opening) } \\
(k N)\end{array}$ & $\begin{array}{l}P(\text { Opening }) \\
(k N)\end{array}$ & $\begin{array}{l}P(A 0) \\
(k N)\end{array}$ & $\begin{array}{l}P(\text { Opening }) \\
(k N)\end{array}$ & $\begin{array}{l}P(\text { Opening }) \\
(k N)\end{array}$ & $\begin{array}{l}P(A 0) \\
(k N)\end{array}$ & $\begin{array}{l}\text { P(Opening }) \\
(k N)\end{array}$ & $\begin{array}{l}P(\text { Opening }) \\
(k N)\end{array}$ \\
\hline & & & & & & & & Offset & Centred & & Offset & Centred & & Offset & Centred \\
\hline 142-N100-MA0.6-FR & 141.82 & 60.63 & 13.66 & 1.27 & 720.0 & 139.27 & 2.45 & 2.31 & 1.66 & 2.22 & 2.12 & 1.5 & 2.03 & 1.96 & 1.38 \\
\hline 142-N120-MA0.6-FR & 142.24 & 60.37 & 13.90 & 1.27 & 740.0 & 139.70 & 2.71 & 2.59 & 1.91 & 2.46 & 2.38 & 1.72 & 2.28 & 2.20 & 1.61 \\
\hline 142-N150-MA0.4-FR & 142.40 & 59.79 & 13.28 & 1.28 & 770.0 & 139.84 & 2.89 & 2.87 & 2.47 & 2.63 & 2.62 & 2.26 & 2.44 & 2.42 & 2.13 \\
\hline 202-N100-MA0.4-FR & 202.04 & 64.79 & 14.78 & 1.38 & 899.2 & 199.28 & 2.44 & 2.31 & 2.05 & 2.29 & 2.15 & 1.90 & 2.13 & 2.03 & 1.76 \\
\hline 202-N100-MA0.6-FR & 202.04 & 64.79 & 14.78 & 1.38 & 899.2 & 199.28 & 2.44 & 2.13 & - & 2.29 & 2.02 & - & 2.13 & 1.95 & - \\
\hline 202-N120-MA0.4-FR & 202.00 & 65.00 & 14.73 & 1.38 & 920.0 & 199.24 & 2.56 & 2.44 & 2.19 & 2.42 & 2.29 & 2.03 & 2.20 & 2.19 & 1.91 \\
\hline 202-N120-MA0.6-FR & 202.00 & 65.00 & 14.73 & 1.38 & 920.0 & 199.24 & 2.56 & 2.27 & 1.72 & 2.42 & 2.16 & 1.60 & 2.20 & 2.10 & 1.49 \\
\hline 202-N150-MA0.4-FR & 202.01 & 65.04 & 14.98 & 1.38 & 950.0 & 199.24 & 2.7 & 2.57 & 2.34 & 2.57 & 2.45 & 2.18 & 4.84 & 2.39 & 3.88 \\
\hline 302-N100-MA0.6-FR & 303.18 & 87.91 & 18.83 & 1.90 & 1200.0 & 299.37 & 4.62 & 4.08 & - & 4.39 & 3.95 & - & 4.30 & 3.89 & - \\
\hline 302-N120-MA0.6-FR & 303.07 & 87.95 & 18.26 & 1.90 & 1221.0 & 299.26 & 4.75 & 4.29 & - & 4.60 & 4.16 & - & 4.52 & 4.11 & - \\
\hline 302-N150-MA0.6-FR & 303.03 & 88.54 & 18.97 & 1.90 & 1249.0 & 299.23 & 5.01 & 4.62 & - & 4.93 & 4.49 & - & 4.84 & 4.45 & - \\
\hline
\end{tabular}


b: For the case of flange fastened to the bearing plate

\begin{tabular}{|c|c|c|c|c|c|c|c|c|c|c|c|c|c|c|c|}
\hline \multirow[t]{2}{*}{ Specimen } & \multirow{2}{*}{$\begin{array}{l}\text { Web } \\
\quad \begin{array}{c}d \\
(\mathrm{~mm})\end{array}\end{array}$} & \multirow{2}{*}{$\begin{array}{c}\text { Flange } \\
\qquad \begin{array}{c}b_{f} \\
(\mathrm{~mm})\end{array}\end{array}$} & \multirow{2}{*}{$\begin{array}{l}\text { Lip } \\
\qquad \begin{array}{c}b_{l} \\
(\mathrm{~mm})\end{array}\end{array}$} & \multirow{2}{*}{$\begin{array}{c}\text { Thickness } \\
t \\
(\mathrm{~mm})\end{array}$} & \multirow{2}{*}{$\begin{array}{c}\text { Length } \\
\qquad \begin{array}{c}L \\
(\mathrm{~mm})\end{array}\end{array}$} & \multirow{2}{*}{$\begin{array}{l}\text { Web } \\
\text { opening } \\
a \\
(\mathrm{~mm})\end{array}$} & \multicolumn{3}{|c|}{ Duplex } & \multicolumn{3}{|c|}{ Ferritic } & \multicolumn{3}{|c|}{ Austenitic } \\
\hline & & & & & & & $\begin{array}{c}P(A 0) \\
(k N)\end{array}$ & $\begin{array}{l}\text { P(Opening) } \\
(k N)\end{array}$ & $\begin{array}{l}\text { P(Opening }) \\
(k N)\end{array}$ & $\begin{array}{l}P(A 0) \\
(k N)\end{array}$ & $\begin{array}{l}\text { P(Opening }) \\
(k N)\end{array}$ & $\begin{array}{l}\text { P(Opening }) \\
(k N)\end{array}$ & $\begin{array}{l}P(A 0) \\
(k N)\end{array}$ & $\begin{array}{l}\text { P(Opening }) \\
(k N)\end{array}$ & $\begin{array}{l}\text { P(Opening }) \\
(k N)\end{array}$ \\
\hline & & & & & & & & Offset & Centred & & Offset & Centred & & Offset & Centred \\
\hline 142-N100-MA0.6-FX & 142.49 & 60.33 & 13.79 & 1.29 & 720.0 & 139.27 & 3.68 & 3.47 & 2.67 & 3.11 & 2.98 & 2.28 & 2.94 & 1.96 & 2.12 \\
\hline 142-N120-MA0.6-FX & 142.38 & 60.21 & 13.68 & 1.29 & 740.0 & 139.70 & 3.77 & 3.58 & 2.82 & 3.22 & 3.11 & 2.42 & 3.05 & 2.20 & 2.28 \\
\hline 142-N150-MA0.4-FX & 142.18 & 60.12 & 13.19 & 1.28 & 770.0 & 139.84 & 4.09 & 4.02 & 3.57 & 3.55 & 3.50 & 3.16 & 3.37 & 2.42 & 3.00 \\
\hline 202-N100-MA0.4-FX & 201.99 & 64.87 & 14.76 & 1.37 & 900.0 & 199.28 & 3.72 & 3.62 & 3.06 & 3.26 & 3.17 & 2.73 & 3.165 & 2.03 & 2.62 \\
\hline 202-N100-MA0.6-FX & 201.99 & 64.87 & 14.76 & 1.37 & 900.0 & 199.28 & 3.72 & 3.40 & - & 3.26 & 3.03 & - & 3.16 & 1.95 & - \\
\hline 202-N120-MA0.4-FX & 202.05 & 64.99 & 14.82 & 1.41 & 920.0 & 199.24 & 3.96 & 3.84 & 3.30 & 3.52 & 3.41 & 2.96 & 3.40 & 2.19 & 2.85 \\
\hline 202-N120-MA0.6-FX & 202.05 & 64.99 & 14.82 & 1.41 & 920.0 & 199.24 & 3.96 & 3.67 & 2.78 & 3.52 & 3.30 & 2.45 & 3.40 & 2.10 & 2.28 \\
\hline 202-N150-MA0.4-FX & 202.00 & 64.93 & 15.00 & 1.41 & 950.0 & 199.24 & 4.31 & 4.18 & 3.67 & 3.89 & 3.79 & 3.32 & 3.76 & 2.39 & 3.24 \\
\hline 302-N100-MA0.6-FX & 303.20 & 88.24 & 18.66 & 1.96 & 1199.0 & 299.37 & 6.53 & 5.93 & - & 5.80 & 5.36 & - & 5.62 & 3.89 & - \\
\hline 302-N120-MA0.6-FX & 303.50 & 88.53 & 18.36 & 1.93 & 1219.0 & 299.26 & 6.93 & 6.40 & - & 6.21 & 5.89 & - & 6.02 & 4.11 & - \\
\hline 302-N150-MA0.6-FX & 303.85 & 88.71 & 18.41 & 1.90 & 1248.3 & 299.23 & 7.55 & 7.05 & - & 6.85 & 6.48 & - & 6.63 & 4.45 & - \\
\hline
\end{tabular}


Table 2: Comparison of numerical results with design strength for the case of flange fastened to the bearing plate without circular web opening

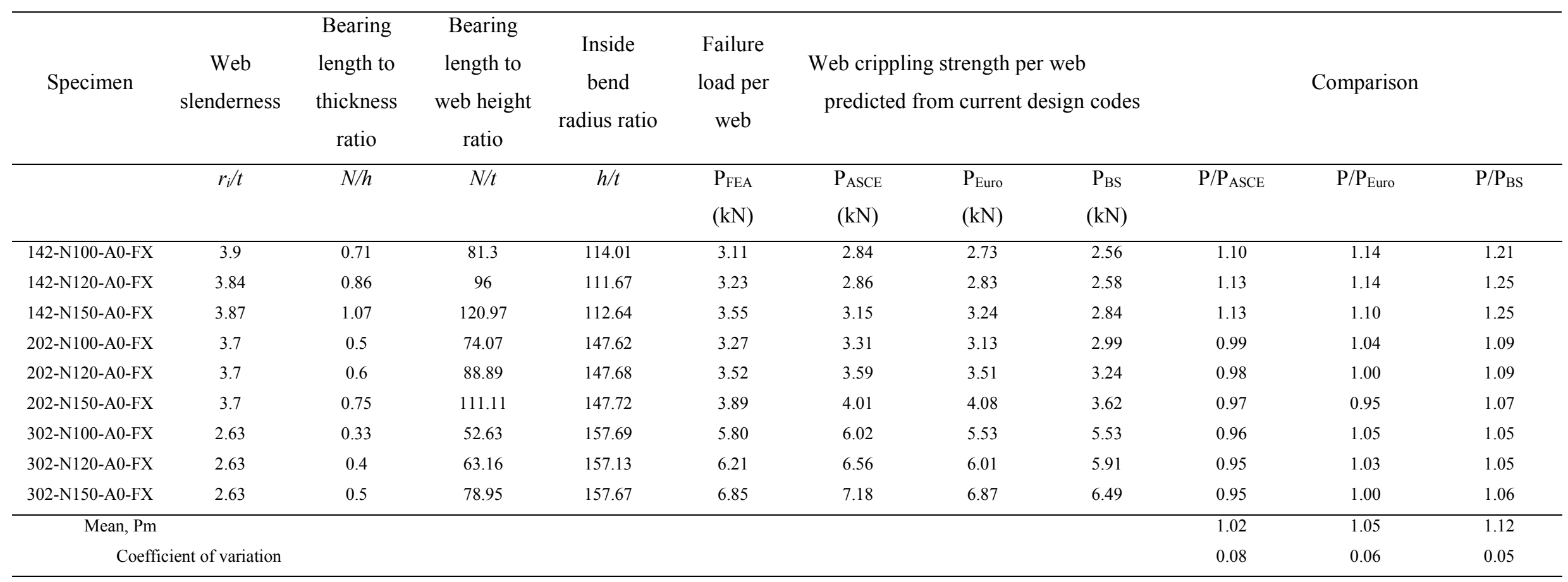


Table 3: Web crippling strengths of duplex stainless steel sections predicted from finite element analysis

a: $a / h$ for centred circular web opening case

\begin{tabular}{|c|c|c|c|c|c|c|c|c|c|c|c|}
\hline \multirow[t]{2}{*}{ Specimen } & \multirow{2}{*}{$\begin{array}{c}\text { Thickness } \\
t \\
(\mathrm{~mm})\end{array}$} & \multicolumn{5}{|c|}{ Unfastened FEA load per web, $\mathrm{P}_{\mathrm{FEA}}$} & \multicolumn{5}{|c|}{ Fastened FEA load per web, $P_{\text {FEA }}$} \\
\hline & & $\begin{array}{l}A(0) \\
(k N)\end{array}$ & $\begin{array}{c}A(0.2) \\
(k N)\end{array}$ & $\begin{array}{c}A(0.4) \\
(k N)\end{array}$ & $\begin{array}{c}A(0.6) \\
(k N)\end{array}$ & $\begin{array}{c}A(0.8) \\
(k N)\end{array}$ & $\begin{array}{l}A(0) \\
(k N)\end{array}$ & $\begin{array}{c}A(0.2) \\
(k N)\end{array}$ & $\begin{array}{c}A(0.4) \\
(k N)\end{array}$ & $\begin{array}{c}A(0.6) \\
(k N)\end{array}$ & $\begin{array}{c}A(0.8) \\
(k N)\end{array}$ \\
\hline 142-N100 & 1.27 & 2.45 & 2.37 & 2.05 & 1.66 & - & 3.68 & 3.55 & 3.07 & 2.67 & - \\
\hline $142-\mathrm{N} 100$ & 4.00 & 21.99 & 21.79 & 20.74 & 20.32 & - & 27.98 & 27.94 & 27.74 & 25.84 & - \\
\hline $142-\mathrm{N} 100$ & 6.00 & 31.36 & 31.33 & 31.16 & 30.27 & - & 34.88 & 34.84 & 34.66 & 33.75 & - \\
\hline $142-\mathrm{N} 120$ & 1.27 & 2.71 & 2.64 & 2.29 & 1.91 & - & 3.77 & 3.63 & 3.23 & 2.82 & - \\
\hline $142-\mathrm{N} 120$ & 4.00 & 20.98 & 20.92 & 20.89 & 20.06 & - & 27.50 & 27.47 & 27.33 & 26.70 & - \\
\hline $142-\mathrm{N} 120$ & 6.00 & 30.87 & 30.74 & 30.59 & 30.09 & - & 34.35 & 34.32 & 34.16 & 33.58 & - \\
\hline $142-\mathrm{N} 150$ & 1.28 & 2.89 & 2.80 & 2.47 & 2.12 & 1.76 & 4.10 & 3.96 & 3.58 & 3.18 & 2.74 \\
\hline $142-\mathrm{N} 150$ & 4.00 & 20.93 & 20.85 & 20.50 & 19.96 & 17.25 & 26.69 & 26.67 & 26.57 & 26.13 & 22.95 \\
\hline 142-N150 & 6.00 & 29.89 & 29.86 & 29.73 & 29.35 & 27.24 & 33.75 & 33.72 & 33.57 & 33.20 & 29.84 \\
\hline 202-N100 & 1.39 & 2.45 & 2.38 & 2.05 & - & - & 3.72 & 3.57 & 3.06 & - & - \\
\hline 202-N100 & 4.00 & 22.46 & 21.89 & 18.06 & - & - & 30.35 & 30.12 & 26.59 & - & - \\
\hline 202-N100 & 6.00 & 32.57 & 32.51 & 31.97 & - & - & 35.86 & 35.79 & 35.46 & - & - \\
\hline 202-N120 & 1.39 & 2.57 & 2.49 & 2.20 & 1.73 & - & 3.97 & 3.80 & 3.31 & 2.78 & - \\
\hline 202-N120 & 4.00 & 22.39 & 22.36 & 19.47 & 14.64 & - & 30.30 & 30.18 & 29.30 & 22.41 & - \\
\hline 202-N120 & 6.00 & 32.32 & 32.27 & 32.00 & 29.37 & - & 35.59 & 35.53 & 35.28 & 33.46 & - \\
\hline $202-N 150$ & 1.39 & 2.70 & 2.62 & 2.34 & 1.92 & - & 4.31 & 4.13 & 3.68 & 3.08 & - \\
\hline 202-N150 & 4.00 & 22.15 & 21.73 & 21.01 & 16.68 & - & 29.76 & 29.68 & 29.32 & 27.55 & - \\
\hline 202-N150 & 6.00 & 31.75 & 31.70 & 31.50 & 30.65 & - & 35.19 & 35.14 & 34.92 & 34.17 & - \\
\hline $302-N 100$ & 1.98 & 4.62 & 4.47 & - & - & - & 6.54 & 6.29 & - & - & - \\
\hline $302-\mathrm{N} 100$ & 4.00 & 21.45 & 20.16 & - & - & - & 30.04 & 28.95 & - & - & - \\
\hline $302-N 100$ & 6.00 & 32.97 & 32.78 & - & - & - & 36.34 & 36.23 & - & - & - \\
\hline $302-\mathrm{N} 120$ & 1.98 & 4.78 & 4.61 & 3.82 & - & - & 6.93 & 6.63 & 5.36 & - & - \\
\hline $302-\mathrm{N} 120$ & 4.00 & 22.24 & 20.91 & 16.82 & - & - & 30.90 & 30.13 & 24.93 & - & - \\
\hline 302-N120 & 6.00 & 32.90 & 32.78 & 31.39 & - & - & 36.17 & 36.09 & 35.36 & - & - \\
\hline $302-N 150$ & 1.99 & 5.02 & 4.89 & 4.00 & - & - & 7.55 & 7.20 & 5.94 & - & - \\
\hline $302-\mathrm{N} 150$ & 4.00 & 23.06 & 21.87 & 17.95 & - & - & 31.16 & 30.78 & 27.71 & - & - \\
\hline $302-\mathrm{N} 150$ & 6.00 & 32.67 & 32.58 & 31.94 & - & - & 35.99 & 35.92 & 35.57 & - & - \\
\hline
\end{tabular}


b: $a / h$ for offset circular web opening case

\begin{tabular}{|c|c|c|c|c|c|c|c|c|c|}
\hline \multirow[t]{2}{*}{ Specimen } & \multirow{2}{*}{$\begin{array}{c}\text { Thickness } \\
T \\
(\mathrm{~mm}) \\
\end{array}$} & \multicolumn{4}{|c|}{ Unfastened FEA load per web, $\mathrm{P}_{\mathrm{FEA}}$} & \multicolumn{4}{|c|}{ Fastened FEA load per web, $\mathrm{P}_{\mathrm{FEA}}$} \\
\hline & & $\begin{array}{l}A(0) \\
(k N) \\
\end{array}$ & $\begin{array}{c}A(0.2) \\
(k N) \\
\end{array}$ & $\begin{array}{c}A(0.4) \\
(k N) \\
\end{array}$ & $\begin{array}{c}A(0.6) \\
(k N) \\
\end{array}$ & $\begin{array}{l}A(0) \\
(k N)\end{array}$ & $\begin{array}{c}A(0.2) \\
(\mathrm{kN}) \\
\end{array}$ & $\begin{array}{c}A(0.4) \\
(k N) \\
\end{array}$ & $\begin{array}{c}A(0.6) \\
(k N)\end{array}$ \\
\hline $142-\mathrm{N} 100$ & 1.27 & 2.45 & 2.45 & 2.40 & 2.32 & 3.68 & 3.66 & 3.58 & 3.47 \\
\hline $142-\mathrm{N} 100$ & 4.00 & 21.97 & 21.93 & 21.72 & 19.95 & 27.98 & 27.94 & 27.62 & 24.44 \\
\hline $142-\mathrm{N} 100$ & 6.00 & 31.37 & 31.31 & 30.99 & 28.81 & 34.88 & 34.76 & 34.48 & 32.60 \\
\hline $142-\mathrm{N} 120$ & 1.27 & 2.73 & 2.72 & 2.68 & 2.60 & 3.77 & 3.76 & 3.69 & 3.59 \\
\hline $142-\mathrm{N} 120$ & 4.00 & 21.72 & 21.68 & 21.46 & 19.51 & 27.50 & 27.46 & 27.11 & 23.87 \\
\hline $142-\mathrm{N} 120$ & 6.00 & 30.78 & 30.71 & 30.38 & 28.07 & 34.35 & 34.29 & 33.95 & 32.02 \\
\hline $142-\mathrm{N} 150$ & 1.28 & 2.90 & 2.90 & 2.87 & 2.77 & 4.10 & 4.08 & 4.03 & 3.94 \\
\hline $142-\mathrm{N} 150$ & 4.00 & 20.94 & 20.90 & 20.65 & 18.53 & 26.69 & 26.66 & 26.23 & 24.41 \\
\hline $142-\mathrm{N} 150$ & 6.00 & 29.89 & 29.83 & 29.46 & 26.90 & 33.75 & 33.68 & 33.34 & 31.28 \\
\hline 202-N100 & 1.39 & 2.45 & 2.42 & 2.32 & 2.14 & 3.72 & 3.71 & 3.62 & 3.41 \\
\hline $202-\mathrm{N} 100$ & 4.00 & 22.46 & 22.34 & 21.90 & 20.44 & 30.35 & 30.27 & 29.92 & 27.82 \\
\hline 202-N100 & 6.00 & 32.57 & 32.48 & 32.09 & 30.75 & 35.86 & 35.76 & 35.36 & 34.06 \\
\hline 202-N120 & 1.39 & 2.57 & 2.53 & 2.44 & 2.28 & 3.97 & 3.95 & 3.85 & 3.68 \\
\hline 202-N120 & 4.00 & 22.39 & 22.28 & 21.86 & 20.71 & 30.30 & 30.23 & 29.86 & 26.11 \\
\hline 202-N120 & 6.00 & 32.32 & 32.23 & 31.85 & 30.47 & 35.59 & 35.50 & 35.10 & 33.80 \\
\hline 202-N150 & 1.39 & 2.70 & 2.67 & 2.57 & 2.44 & 4.31 & 4.29 & 4.19 & 4.06 \\
\hline $202-\mathrm{N} 150$ & 4.00 & 22.15 & 22.06 & 21.77 & 20.57 & 29.76 & 29.69 & 29.32 & 26.84 \\
\hline 202-N150 & 6.00 & 31.75 & 31.66 & 31.29 & 29.80 & 35.19 & 35.10 & 34.71 & 33.39 \\
\hline $302-\mathrm{N} 100$ & 1.98 & 4.62 & 4.62 & 4.40 & 4.08 & 6.54 & 6.41 & 6.19 & 5.94 \\
\hline $302-\mathrm{N} 100$ & 2.00 & 21.45 & 21.22 & 20.65 & 19.82 & 30.04 & 29.93 & 29.56 & 28.62 \\
\hline $302-\mathrm{N} 100$ & 4.00 & 32.97 & 32.85 & 32.39 & 31.08 & 36.34 & 36.24 & 35.80 & 34.50 \\
\hline $302-\mathrm{N} 120$ & 1.98 & 4.78 & 4.78 & 4.57 & 4.30 & 6.93 & 6.81 & 6.63 & 6.41 \\
\hline $302-\mathrm{N} 120$ & 2.00 & 22.24 & 22.03 & 21.50 & 20.63 & 30.90 & 30.79 & 30.39 & 29.20 \\
\hline $302-\mathrm{N} 120$ & 4.00 & 32.90 & 32.79 & 32.33 & 31.00 & 36.17 & 36.07 & 35.64 & 34.33 \\
\hline $302-\mathrm{N} 150$ & 1.99 & 5.09 & 5.05 & 4.89 & 4.63 & 7.55 & 7.47 & 7.31 & 7.06 \\
\hline $302-\mathrm{N} 150$ & 2.00 & 23.06 & 22.90 & 22.39 & 21.38 & 31.16 & 31.05 & 30.63 & 29.30 \\
\hline $302-N 150$ & 4.00 & 32.67 & 32.56 & 32.11 & 30.78 & 35.99 & 35.90 & 35.47 & 34.18 \\
\hline
\end{tabular}


c: $x / h$ for offset circular web opening case

\begin{tabular}{|c|c|c|c|c|c|c|c|c|c|}
\hline \multirow[t]{2}{*}{ Specimen } & \multirow{2}{*}{$\begin{array}{c}\text { Thickness } \\
t \\
(\mathrm{~mm}) \\
\end{array}$} & \multicolumn{4}{|c|}{ Unfastened FEA load per web, $P($ FEA $)$} & \multicolumn{4}{|c|}{ Fastened FEA load per web, $\mathrm{P}_{\mathrm{FEA}}$} \\
\hline & & $\begin{array}{l}X(0) \\
(k N)\end{array}$ & $\begin{array}{c}X(0.2) \\
(k N)\end{array}$ & $\begin{array}{c}X(0.4) \\
(k N)\end{array}$ & $\begin{array}{c}X(0.6) \\
(k N)\end{array}$ & $\begin{array}{l}X(0) \\
(k N)\end{array}$ & $\begin{array}{c}X(0.2) \\
(k N)\end{array}$ & $\begin{array}{c}X(0.4) \\
(k N)\end{array}$ & $\begin{array}{c}X(0.6) \\
(k N)\end{array}$ \\
\hline $142-\mathrm{N} 100-\mathrm{A} 0$ & 1.27 & 2.38 & 2.38 & 2.38 & 2.38 & 3.63 & 3.63 & 3.63 & 3.63 \\
\hline $142-\mathrm{N} 100-\mathrm{A} 0.2$ & 1.27 & 2.33 & 2.34 & 2.35 & 2.36 & 3.57 & 3.58 & 3.60 & 3.62 \\
\hline $142-\mathrm{N} 100-\mathrm{A} 0.4$ & 1.27 & 2.18 & 2.22 & 2.26 & 2.29 & 3.39 & 3.44 & 3.50 & 3.53 \\
\hline 142-N100-A0.6 & 1.27 & 1.99 & 2.07 & 2.14 & 2.20 & 3.16 & 3.24 & 3.32 & 3.37 \\
\hline $142-\mathrm{N} 100-\mathrm{A} 0.8$ & 1.27 & --- & --- & --- & --- & --- & --- & --- & --- \\
\hline $142-\mathrm{N} 120-\mathrm{A} 0$ & 1.27 & 2.68 & 2.68 & 2.68 & 2.68 & 3.74 & 3.74 & 3.74 & 3.74 \\
\hline 142-N120-A0.2 & 1.27 & 2.63 & 2.64 & 2.65 & 2.63 & 3.68 & 3.69 & 3.71 & 3.73 \\
\hline 142-N120-A0.4 & 1.27 & 2.39 & 2.43 & 2.47 & 2.39 & 3.51 & 3.56 & 3.61 & 3.63 \\
\hline 142-N120-A0.6 & 1.27 & 2.22 & 2.29 & 2.36 & 2.22 & 3.29 & 3.37 & 3.43 & 3.47 \\
\hline 142-N120-A0.8 & 1.27 & --- & --- & --- & --- & --- & --- & --- & --- \\
\hline $142-\mathrm{N} 150-\mathrm{A} 0$ & 1.28 & 2.74 & 2.74 & 2.74 & 2.74 & 4.07 & 4.07 & 4.07 & 4.07 \\
\hline $142-\mathrm{N} 150-\mathrm{A} 0.2$ & 1.28 & 2.69 & 2.70 & 2.70 & 2.71 & 4.01 & 4.02 & 4.04 & 4.06 \\
\hline $142-\mathrm{N} 150-\mathrm{A} 0.4$ & 1.28 & 2.56 & 2.60 & 2.62 & 2.65 & 3.86 & 3.91 & 3.94 & 3.96 \\
\hline 142-N150-A0.6 & 1.28 & 2.42 & 2.47 & 2.53 & 2.57 & 3.66 & 3.71 & 3.75 & 3.79 \\
\hline 142-N150-A0.8 & 1.28 & 2.38 & 2.47 & 2.53 & 2.55 & --- & --- & --- & --- \\
\hline 202-N100-A0 & 1.39 & 2.26 & 2.26 & 2.26 & 2.26 & 3.72 & 3.72 & 3.72 & 3.72 \\
\hline 202-N100-A0.2 & 1.39 & 2.21 & 2.22 & 2.22 & 2.37 & 3.63 & 3.64 & 3.68 & 3.71 \\
\hline 202-N100-A0.4 & 1.39 & 2.05 & 2.17 & 2.23 & 2.26 & 3.45 & 3.54 & 3.55 & 3.61 \\
\hline 202-N100-A0.6 & 1.39 & 1.81 & 1.86 & 1.92 & 1.98 & 3.08 & 3.21 & 3.33 & 3.38 \\
\hline 202-N120-A0 & 1.39 & 2.38 & 2.38 & 2.38 & 2.38 & 3.96 & 3.96 & 3.96 & 3.96 \\
\hline 202-N120-A0.2 & 1.39 & 2.28 & 2.28 & 2.29 & 2.42 & 3.67 & 3.71 & 3.93 & 3.96 \\
\hline 202-N120-A0.4 & 1.39 & 2.16 & 2.19 & 2.21 & 2.37 & 3.65 & 3.74 & 3.80 & 3.85 \\
\hline 202-N120-A0.6 & 1.39 & 1.92 & 2.07 & 2.07 & 2.22 & 3.39 & 3.49 & 3.56 & 3.61 \\
\hline 202-N150-A0 & 1.45 & 2.51 & 2.51 & 2.51 & 2.51 & 4.33 & 4.33 & 4.33 & 4.33 \\
\hline 202-N150-A0.2 & 1.45 & 2.46 & 2.47 & 2.47 & 2.60 & 4.26 & 4.29 & 4.32 & 4.34 \\
\hline 202-N150-A0.4 & 1.45 & 2.30 & 2.32 & 2.35 & 2.50 & 4.08 & 4.15 & 4.17 & 4.19 \\
\hline 202-N150-A0.6 & 1.45 & 2.11 & 2.19 & 2.25 & 2.37 & 3.80 & 3.88 & 3.93 & 4.01 \\
\hline $302-\mathrm{N} 100-\mathrm{A} 0$ & 1.98 & 4.05 & 4.05 & 4.05 & 4.05 & 6.52 & 6.52 & 6.52 & 6.52 \\
\hline $302-\mathrm{N} 100-\mathrm{A} 0.2$ & 1.98 & 3.95 & 3.97 & 4.01 & 4.05 & 6.35 & 6.49 & 6.50 & 6.54 \\
\hline 302-N120-A0 & 1.96 & 4.21 & 4.21 & 4.21 & 4.21 & 6.90 & 6.90 & 6.90 & 6.90 \\
\hline 302-N120-A0.2 & 1.96 & 4.14 & 4.18 & 4.22 & 4.23 & 6.71 & 6.78 & 6.85 & 6.89 \\
\hline 302-N120-A0.4 & 1.96 & 3.83 & 3.97 & 4.04 & 4.05 & 6.45 & 6.60 & 6.65 & 6.67 \\
\hline 302-N120-A0.6 & 1.96 & 3.38 & 3.61 & 3.72 & 3.78 & --- & --- & --- & --- \\
\hline $302-\mathrm{N} 150-\mathrm{A} 0$ & 1.99 & 4.53 & 4.53 & 4.53 & 4.53 & 7.88 & 7.88 & 7.88 & 7.88 \\
\hline $302-\mathrm{N} 150-\mathrm{A} 0.2$ & 1.99 & 4.43 & 4.48 & 4.51 & 4.50 & 7.58 & 7.60 & 7.64 & 7.67 \\
\hline 302-N150-A0.4 & 1.99 & 4.11 & 4.24 & 4.31 & 4.32 & 7.19 & 7.24 & 7.26 & 7.41 \\
\hline 302-N150-A0.6 & 1.99 & 3.68 & 3.89 & 3.99 & 4.06 & --- & --- & --- & --- \\
\hline
\end{tabular}


Table 4: Web crippling strengths of austenitic stainless steel sections predicted from finite element analysis

a: $a / h$ for centred circular web opening case

\begin{tabular}{|c|c|c|c|c|c|c|c|c|c|c|c|}
\hline \multirow[t]{2}{*}{ Specimen } & \multirow{2}{*}{$\begin{array}{c}\text { Thickness } \\
t \\
(\mathrm{~mm})\end{array}$} & \multicolumn{5}{|c|}{ Unfastened FEA load per web, $\mathrm{P}_{\mathrm{FEA}}$} & \multicolumn{5}{|c|}{ Fastened FEA load per web, $\mathrm{P}_{\mathrm{FEA}}$} \\
\hline & & $\begin{array}{l}A(0) \\
(k N)\end{array}$ & $\begin{array}{c}A(0.2) \\
(k N)\end{array}$ & $\begin{array}{c}A(0.4) \\
(k N)\end{array}$ & $\begin{array}{c}A(0.6) \\
(k N)\end{array}$ & $\begin{array}{c}A(0.8) \\
(k N)\end{array}$ & $\begin{array}{l}A(0) \\
(k N)\end{array}$ & $\begin{array}{c}A(0.2) \\
(k N)\end{array}$ & $\begin{array}{c}A(0.4) \\
(k N)\end{array}$ & $\begin{array}{c}A(0.6) \\
(k N)\end{array}$ & $\begin{array}{c}A(0.8) \\
(k N)\end{array}$ \\
\hline $142-\mathrm{N} 100$ & 1.27 & 1.73 & 1.39 & - & 2.94 & 2.91 & 2.54 & 2.13 & - & 2.04 & 1.99 \\
\hline $142-\mathrm{N} 100$ & 4.00 & 15.56 & 13.07 & - & 21.50 & 21.47 & 21.29 & 19.28 & - & 16.11 & 16.06 \\
\hline $142-\mathrm{N} 100$ & 6.00 & 25.32 & 24.91 & - & 31.03 & 31.00 & 30.85 & 29.87 & - & 25.50 & 25.45 \\
\hline $142-\mathrm{N} 120$ & 1.27 & 1.94 & 1.61 & - & 3.05 & 2.99 & 2.66 & 2.28 & - & 2.28 & 2.22 \\
\hline $142-\mathrm{N} 120$ & 4.00 & 15.64 & 14.42 & - & 21.15 & 21.13 & 21.02 & 20.58 & - & 15.87 & 15.83 \\
\hline $142-\mathrm{N} 120$ & 6.00 & 24.90 & 24.69 & - & 30.53 & 30.50 & 30.39 & 29.69 & - & 25.02 & 24.99 \\
\hline $142-\mathrm{N} 150$ & 1.28 & 2.13 & 1.82 & 1.51 & 3.37 & 3.28 & 3.01 & 2.66 & 2.26 & 2.45 & 2.38 \\
\hline $142-\mathrm{N} 150$ & 4.00 & 15.18 & 14.86 & 13.32 & 20.53 & 20.51 & 20.44 & 20.17 & 18.28 & 15.30 & 15.27 \\
\hline $142-\mathrm{N} 150$ & 6.00 & 24.09 & 23.91 & 22.66 & 29.80 & 29.78 & 29.68 & 29.14 & 26.14 & 24.20 & 24.17 \\
\hline 202-N100 & 1.39 & 1.76 & - & - & 3.17 & 3.10 & 2.62 & - & - & 2.13 & 2.09 \\
\hline 202-N100 & 4.00 & 14.33 & - & - & 24.96 & 24.62 & 20.77 & - & - & 17.78 & 17.27 \\
\hline 202-N100 & 6.00 & 28.51 & - & - & 34.08 & 34.03 & 33.72 & - & - & 28.87 & 28.85 \\
\hline 202-N120 & 1.39 & 1.91 & 1.50 & - & 3.41 & 3.31 & 2.86 & 2.29 & - & 2.26 & 2.21 \\
\hline $202-\mathrm{N} 120$ & 4.00 & 15.41 & 11.41 & - & 24.96 & 24.85 & 22.94 & 17.46 & - & 18.20 & 17.85 \\
\hline 202-N120 & 6.00 & 28.79 & 24.53 & - & 33.87 & 33.83 & 33.62 & 30.09 & - & 28.83 & 28.84 \\
\hline 202-N150 & 1.39 & 2.09 & 1.68 & - & 3.76 & 3.65 & 3.24 & 2.64 & - & 2.44 & 2.37 \\
\hline $202-\mathrm{N} 150$ & 4.00 & 16.59 & 13.32 & - & 24.41 & 24.35 & 24.09 & 21.35 & - & 18.00 & 17.84 \\
\hline $202-\mathrm{N} 150$ & 6.00 & 28.14 & 28.04 & - & 33.34 & 33.30 & 33.13 & 32.14 & - & 28.16 & 28.16 \\
\hline $302-\mathrm{N} 100$ & 1.98 & - & - & - & 5.62 & 5.53 & - & - & - & 4.31 & 4.20 \\
\hline $302-\mathrm{N} 100$ & 4.00 & - & - & - & 24.72 & 24.49 & - & - & - & 18.63 & 17.79 \\
\hline $302-\mathrm{N} 100$ & 6.00 & - & - & - & 35.65 & 35.55 & - & - & - & 32.05 & 31.81 \\
\hline $302-\mathrm{N} 120$ & 1.98 & 3.57 & - & - & 6.02 & 5.88 & 4.66 & - & - & 4.53 & 4.39 \\
\hline $302-\mathrm{N} 120$ & 4.00 & 14.46 & - & - & 26.79 & 26.14 & 20.54 & - & - & 19.46 & 18.51 \\
\hline $302-\mathrm{N} 120$ & 6.00 & 29.86 & - & - & 35.60 & 35.51 & 34.64 & - & - & 32.08 & 31.96 \\
\hline $302-\mathrm{N} 150$ & 1.99 & 3.89 & - & - & 6.64 & 6.45 & 5.31 & - & - & 4.85 & 4.69 \\
\hline $302-\mathrm{N} 150$ & 4.00 & 15.58 & - & - & 28.54 & 27.98 & 23.09 & - & - & 20.56 & 19.48 \\
\hline $302-\mathrm{N} 150$ & 6.00 & 30.75 & - & - & 35.99 & 35.92 & 35.57 & - & - & 31.88 & 31.81 \\
\hline
\end{tabular}


b: $a / h$ for offset circular web opening case

\begin{tabular}{|c|c|c|c|c|c|c|c|c|c|}
\hline \multirow[t]{2}{*}{ Specimen } & \multirow{2}{*}{$\begin{array}{c}\text { Thickness } \\
t \\
(\mathrm{~mm})\end{array}$} & \multicolumn{4}{|c|}{ Unfastened FEA load per web, $P_{\text {FEA }}$} & \multicolumn{4}{|c|}{ Fastened FEA load per web, $P_{\text {FEA }}$} \\
\hline & & $\begin{array}{l}A(0) \\
(k N)\end{array}$ & $\begin{array}{c}A(0.2) \\
(k N)\end{array}$ & $\begin{array}{c}A(0.4) \\
(k N)\end{array}$ & $\begin{array}{c}A(0.6) \\
(k N)\end{array}$ & $\begin{array}{l}A(0) \\
(k N) \\
\end{array}$ & $\begin{array}{c}A(0.2) \\
(k N)\end{array}$ & $\begin{array}{c}A(0.4) \\
(k N)\end{array}$ & $\begin{array}{c}A(0.6) \\
(k N)\end{array}$ \\
\hline $142-\mathrm{N} 100$ & 1.27 & 2.04 & 2.03 & 2.01 & 1.96 & - & 2.93 & 2.89 & 2.83 \\
\hline $142-\mathrm{N} 100$ & 4.00 & 16.11 & 16.07 & 15.89 & 14.37 & - & 21.46 & 21.07 & 18.15 \\
\hline $142-\mathrm{N} 100$ & 6.00 & 25.50 & 25.43 & 25.09 & 23.87 & - & 30.99 & 30.60 & 27.04 \\
\hline $142-\mathrm{N} 120$ & 1.27 & 2.28 & 2.28 & 2.25 & 2.21 & - & 3.05 & 3.01 & 2.95 \\
\hline $142-\mathrm{N} 120$ & 4.00 & 15.87 & 15.84 & 15.64 & 14.05 & - & 21.12 & 20.67 & 17.73 \\
\hline $142-\mathrm{N} 120$ & 6.00 & 25.02 & 24.97 & 24.58 & 21.72 & - & 30.49 & 30.07 & 26.49 \\
\hline $142-\mathrm{N} 150$ & 1.28 & 2.45 & 2.44 & 2.43 & 2.36 & 3.37 & 3.37 & 3.33 & 3.21 \\
\hline $142-\mathrm{N} 150$ & 4.00 & 15.30 & 15.27 & 15.03 & 13.33 & 20.53 & 20.49 & 19.94 & 16.99 \\
\hline $142-\mathrm{N} 150$ & 6.00 & 24.20 & 24.14 & 23.68 & 20.70 & 29.80 & 29.77 & 29.23 & 25.60 \\
\hline 202-N100 & 1.39 & 2.13 & 2.11 & 2.04 & - & - & 3.14 & 3.06 & 2.95 \\
\hline $202-\mathrm{N} 100$ & 4.00 & 17.78 & 17.72 & 17.50 & - & - & 24.92 & 24.64 & 21.60 \\
\hline $202-\mathrm{N} 100$ & 6.00 & 28.87 & 28.81 & 28.52 & - & - & 34.01 & 33.70 & 31.05 \\
\hline $202-\mathrm{N} 120$ & 1.39 & 2.26 & 2.24 & 2.20 & 2.11 & - & 3.38 & 3.30 & 3.23 \\
\hline $202-\mathrm{N} 120$ & 4.00 & 18.20 & 18.14 & 17.91 & 16.61 & - & 24.92 & 24.60 & 21.34 \\
\hline $202-\mathrm{N} 120$ & 6.00 & 28.83 & 28.77 & 28.45 & 25.94 & - & 33.81 & 33.48 & 30.73 \\
\hline $202-\mathrm{N} 150$ & 1.39 & 2.44 & 2.43 & 2.39 & 2.29 & - & 3.74 & 3.69 & 3.61 \\
\hline $202-\mathrm{N} 150$ & 4.00 & 18.00 & 17.94 & 17.71 & 16.11 & - & 24.37 & 24.00 & 20.57 \\
\hline $202-\mathrm{N} 150$ & 6.00 & 28.16 & 28.10 & 27.76 & 24.98 & - & 33.28 & 32.94 & 29.97 \\
\hline $302-\mathrm{N} 100$ & 1.98 & 4.31 & 4.28 & - & - & - & 5.56 & 5.41 & - \\
\hline $302-\mathrm{N} 100$ & 2.00 & 18.63 & 18.48 & - & - & - & 24.67 & 24.54 & - \\
\hline $302-\mathrm{N} 100$ & 4.00 & 32.05 & 31.94 & - & - & - & 35.56 & 35.15 & - \\
\hline $302-\mathrm{N} 120$ & 1.98 & 4.53 & 4.49 & 4.35 & - & - & 5.97 & 5.84 & 5.70 \\
\hline $302-\mathrm{N} 120$ & 2.00 & 19.46 & 19.33 & 18.97 & - & - & 26.79 & 26.67 & 25.92 \\
\hline $302-\mathrm{N} 120$ & 4.00 & 32.08 & 31.98 & 31.56 & - & - & 35.50 & 35.10 & 33.74 \\
\hline $302-\mathrm{N} 150$ & 1.99 & 5.23 & 5.05 & 4.68 & - & - & 6.60 & 6.51 & 6.35 \\
\hline $302-\mathrm{N} 150$ & 2.00 & 20.56 & 20.44 & 20.10 & - & - & 28.46 & 28.16 & 26.24 \\
\hline $302-\mathrm{N} 150$ & 4.00 & 31.88 & 31.78 & 31.37 & - & - & 35.30 & 34.90 & 33.55 \\
\hline
\end{tabular}


c: $x / h$ for offset circular web opening case

\begin{tabular}{|c|c|c|c|c|c|c|c|c|c|}
\hline \multirow[t]{2}{*}{ Specimen } & \multirow{2}{*}{$\begin{array}{c}\text { Thickness } \\
t \\
(\mathrm{~mm}) \\
\end{array}$} & \multicolumn{4}{|c|}{ Unfastened FEA load per web, $P(F E A)$} & \multicolumn{4}{|c|}{ Fastened FEA load per web, $\mathrm{P}_{\mathrm{FEA}}$} \\
\hline & & $\begin{array}{l}X(0) \\
(k N)\end{array}$ & $\begin{array}{c}X(0.2) \\
(k N)\end{array}$ & $\begin{array}{c}X(0.4) \\
(k N)\end{array}$ & $\begin{array}{c}X(0.6) \\
(k N)\end{array}$ & $\begin{array}{l}X(0) \\
(k N)\end{array}$ & $\begin{array}{c}X(0.2) \\
(k N)\end{array}$ & $\begin{array}{c}X(0.4) \\
(k N)\end{array}$ & $\begin{array}{c}X(0.6) \\
(k N)\end{array}$ \\
\hline $142-\mathrm{N} 100-\mathrm{A} 0$ & 1.27 & 2.14 & 2.14 & 2.14 & 2.14 & 2.01 & 2.01 & 2.01 & 2.01 \\
\hline $142-\mathrm{N} 100-\mathrm{A} 0.2$ & 1.27 & 2.10 & 2.11 & 2.12 & 2.13 & 1.97 & 1.98 & 1.99 & 2.00 \\
\hline $142-\mathrm{N} 100-\mathrm{A} 0.4$ & 1.27 & 1.98 & 2.03 & 2.06 & 2.09 & 1.86 & 1.90 & 1.93 & 1.96 \\
\hline 142-N100-A0.6 & 1.27 & 1.84 & 1.92 & 1.99 & 2.04 & 1.72 & 1.80 & 1.86 & 1.90 \\
\hline 142-N100-A0.8 & 1.27 & --- & --- & --- & --- & --- & --- & --- & --- \\
\hline 142-N120-A0 & 1.27 & 2.31 & 2.31 & 2.31 & 2.31 & 2.23 & 2.23 & 2.23 & 2.23 \\
\hline $142-\mathrm{N} 120-\mathrm{A} 0.2$ & 1.27 & 2.27 & 2.28 & 2.29 & 2.30 & 2.20 & 2.21 & 2.22 & 2.22 \\
\hline 142-N120-A0.4 & 1.27 & 2.16 & 2.20 & 2.23 & 2.26 & 2.09 & 2.13 & 2.16 & 2.19 \\
\hline 142-N120-A0.6 & 1.27 & 2.03 & 2.11 & 2.16 & 2.20 & 1.97 & 2.04 & 2.09 & 2.13 \\
\hline 142-N120-A0.8 & 1.27 & --- & --- & --- & --- & --- & --- & --- & --- \\
\hline $142-\mathrm{N} 150-\mathrm{A} 0$ & 1.28 & 2.54 & 2.54 & 2.54 & 2.54 & 2.38 & 2.38 & 2.38 & 2.38 \\
\hline $142-\mathrm{N} 150-\mathrm{A} 0.2$ & 1.28 & 2.51 & 2.51 & 2.52 & 2.53 & 2.35 & 2.36 & 2.36 & 2.37 \\
\hline $142-\mathrm{N} 150-\mathrm{A} 0.4$ & 1.28 & 2.42 & 2.45 & 2.48 & 2.50 & 2.27 & 2.30 & 2.32 & 2.34 \\
\hline 142-N150-A0.6 & 1.28 & 2.30 & 2.36 & 2.41 & 2.44 & 2.16 & 2.21 & 2.26 & 2.29 \\
\hline 142-N150-A0.8 & 1.28 & 2.14 & 2.19 & 2.19 & 2.24 & 2.01 & 2.06 & 2.07 & 2.08 \\
\hline 202-N100-A0 & 1.39 & 2.28 & 2.28 & 2.28 & 2.28 & 2.11 & 2.11 & 2.11 & 2.11 \\
\hline 202-N100-A0.2 & 1.39 & 2.07 & 2.20 & 2.21 & 2.22 & 2.07 & 2.07 & 2.08 & 2.08 \\
\hline 202-N100-A0.4 & 1.39 & 1.90 & 2.06 & 2.09 & 2.11 & 1.90 & 1.93 & 1.98 & 2.01 \\
\hline 202-N100-A0.6 & 1.39 & 1.69 & 1.79 & 1.87 & 1.88 & --- & --- & --- & --- \\
\hline 202-N120-A0 & 1.39 & 2.36 & 2.36 & 2.36 & 2.36 & 2.38 & 2.38 & 2.38 & 2.38 \\
\hline 202-N120-A0.2 & 1.39 & 2.14 & 2.15 & 2.28 & 2.29 & 2.35 & 2.36 & 2.36 & 2.37 \\
\hline 202-N120-A0.4 & 1.39 & 2.05 & 2.09 & 2.21 & 2.24 & 2.27 & 2.30 & 2.32 & 2.34 \\
\hline 202-N120-A0.6 & 1.39 & 1.86 & 1.95 & 2.06 & 2.11 & 2.16 & 2.21 & 2.26 & 2.29 \\
\hline 202-N150-A0 & 1.45 & 2.59 & 2.59 & 2.59 & 2.59 & 2.01 & 2.06 & 2.07 & 2.08 \\
\hline 202-N150-A0.2 & 1.45 & 2.35 & 2.36 & 2.46 & 2.47 & 2.11 & 2.11 & 2.11 & 2.11 \\
\hline 202-N150-A0.4 & 1.45 & 2.23 & 2.27 & 2.37 & 2.38 & 2.07 & 2.07 & 2.08 & 2.08 \\
\hline 202-N150-A0.6 & 1.45 & 2.07 & 2.14 & 2.23 & 2.26 & 1.90 & 1.93 & 1.98 & 2.01 \\
\hline $302-\mathrm{N} 100-\mathrm{A} 0$ & 1.98 & 4.64 & 4.64 & 4.64 & 4.64 & 2.23 & 2.23 & 2.23 & 2.23 \\
\hline 302-N100-A0.2 & 1.98 & 4.54 & 4.57 & 4.61 & 4.61 & 2.19 & 2.19 & 2.20 & 2.21 \\
\hline 302-N120-A0 & 1.96 & 4.76 & 4.76 & 4.76 & 4.76 & 2.05 & 2.09 & 2.13 & 2.16 \\
\hline 302-N120-A0.2 & 1.96 & 4.66 & 4.70 & 4.73 & 4.74 & 2.38 & 2.38 & 2.38 & 2.38 \\
\hline 302-N120-A0.4 & 1.96 & 4.29 & 4.43 & 4.50 & 4.52 & 4.05 & 4.20 & 4.29 & 4.31 \\
\hline 302-N120-A0.6 & 1.96 & 3.84 & 4.06 & 4.19 & 4.30 & --- & --- & --- & --- \\
\hline $302-\mathrm{N} 150-\mathrm{A} 0$ & 1.99 & 5.24 & 5.24 & 5.24 & 5.24 & 4.80 & 4.80 & 4.80 & 4.80 \\
\hline 302-N150-A0.2 & 1.99 & 5.13 & 5.17 & 5.21 & 5.21 & --- & --- & --- & --- \\
\hline 302-N150-A0.4 & 1.99 & 4.77 & 4.92 & 5.02 & 5.05 & --- & --- & --- & --- \\
\hline $302-\mathrm{N} 150-\mathrm{A} 0.6$ & 1.99 & 4.36 & 4.57 & 4.70 & 4.82 & --- & --- & --- & --- \\
\hline
\end{tabular}


Table 5: Web crippling strengths of ferritic stainless steel sections predicted from finite element analysis

a: $a / h$ for centred circular web opening case

\begin{tabular}{|c|c|c|c|c|c|c|c|c|c|c|c|}
\hline \multirow[t]{2}{*}{ Specimen } & \multirow{2}{*}{$\begin{array}{c}\text { Thickness } \\
t \\
(\mathrm{~mm})\end{array}$} & \multicolumn{5}{|c|}{ Unfastened FEA load per web, $\mathrm{P}_{\mathrm{FEA}}$} & \multicolumn{5}{|c|}{ Fastened FEA load per web, $P_{\text {FEA }}$} \\
\hline & & $\begin{array}{l}A(0) \\
(k N)\end{array}$ & $\begin{array}{c}A(0.2) \\
(k N)\end{array}$ & $\begin{array}{c}A(0.4) \\
(k N)\end{array}$ & $\begin{array}{c}A(0.6) \\
(k N)\end{array}$ & $\begin{array}{c}A(0.8) \\
(k N)\end{array}$ & $\begin{array}{l}A(0) \\
(\mathrm{kN})\end{array}$ & $\begin{array}{c}A(0.2) \\
(k N)\end{array}$ & $\begin{array}{c}A(0.4) \\
(k N)\end{array}$ & $\begin{array}{c}A(0.6) \\
(k N)\end{array}$ & $\begin{array}{c}A(0.8) \\
(k N)\end{array}$ \\
\hline $142-\mathrm{N} 100$ & 1.27 & 2.23 & 2.17 & 1.85 & 1.50 & - & 3.11 & 3.07 & 2.68 & 2.28 & - \\
\hline $142-\mathrm{N} 100$ & 4.00 & 18.33 & 18.30 & 17.70 & 14.70 & - & 23.29 & 23.27 & 23.12 & 20.11 & - \\
\hline $142-\mathrm{N} 100$ & 6.00 & 28.33 & 28.30 & 28.23 & 27.83 & - & 33.31 & 33.28 & 33.12 & 32.09 & - \\
\hline $142-\mathrm{N} 120$ & 1.27 & 2.47 & 2.39 & 2.06 & 1.73 & - & 3.23 & 3.14 & 2.80 & 2.42 & - \\
\hline $142-\mathrm{N} 120$ & 4.00 & 18.06 & 18.04 & 17.88 & 16.25 & - & 22.96 & 22.94 & 22.86 & 22.49 & - \\
\hline $142-\mathrm{N} 120$ & 6.00 & 27.78 & 27.77 & 27.71 & 27.52 & - & 32.80 & 32.77 & 32.64 & 32.00 & - \\
\hline $142-\mathrm{N} 150$ & 1.28 & 2.63 & 2.54 & 2.26 & 1.93 & 1.60 & 3.55 & 3.46 & 3.17 & 2.79 & 2.40 \\
\hline $142-\mathrm{N} 150$ & 4.00 & 17.39 & 17.38 & 17.33 & 16.95 & 14.92 & 22.32 & 22.31 & 22.25 & 22.08 & 19.79 \\
\hline $142-\mathrm{N} 150$ & 6.00 & 26.92 & 26.90 & 26.84 & 26.67 & 24.99 & 32.11 & 32.08 & 31.97 & 31.46 & 27.68 \\
\hline $202-\mathrm{N} 100$ & 1.39 & 2.29 & 2.23 & 1.90 & - & - & 3.27 & 3.10 & 2.73 & - & - \\
\hline $202-\mathrm{N} 100$ & 4.00 & 19.68 & 19.30 & 15.98 & - & - & 27.05 & 26.62 & 21.95 & - & - \\
\hline $202-\mathrm{N} 100$ & 6.00 & 31.10 & 31.08 & 30.62 & - & - & 35.32 & 35.26 & 34.91 & - & - \\
\hline 202-N120 & 1.39 & 2.43 & 2.36 & 2.04 & 1.61 & - & 3.52 & 3.41 & 2.97 & 2.45 & - \\
\hline $202-\mathrm{N} 120$ & 4.00 & 20.15 & 19.64 & 17.21 & 12.82 & - & 27.22 & 27.08 & 24.58 & 18.77 & - \\
\hline $202-\mathrm{N} 120$ & 6.00 & 30.92 & 30.91 & 30.80 & 27.32 & - & 35.11 & 35.06 & 34.80 & 31.78 & - \\
\hline $202-\mathrm{N} 150$ & 1.39 & 2.57 & 2.50 & 2.19 & 1.78 & - & 3.89 & 3.76 & 3.33 & 2.76 & - \\
\hline $202-\mathrm{N} 150$ & 4.00 & 20.03 & 19.78 & 18.54 & 14.76 & - & 26.74 & 26.67 & 26.34 & 22.93 & - \\
\hline $202-\mathrm{N} 150$ & 6.00 & 30.29 & 30.27 & 30.19 & 29.49 & - & 34.66 & 34.61 & 34.39 & 33.58 & - \\
\hline $302-\mathrm{N} 100$ & 1.98 & 4.40 & 4.27 & - & - & - & 5.80 & 5.65 & - & - & - \\
\hline $302-\mathrm{N} 100$ & 4.00 & 19.97 & 18.89 & - & - & - & 26.13 & 25.71 & - & - & - \\
\hline $302-\mathrm{N} 100$ & 6.00 & 32.38 & 32.19 & - & - & - & 35.96 & 35.85 & - & - & - \\
\hline $302-\mathrm{N} 120$ & 1.98 & 4.61 & 4.45 & 3.64 & - & - & 6.21 & 6.02 & 4.88 & - & - \\
\hline $302-\mathrm{N} 120$ & 4.00 & 20.81 & 19.62 & 15.59 & - & - & 28.17 & 27.19 & 21.67 & - & - \\
\hline $302-\mathrm{N} 120$ & 6.00 & 32.38 & 32.26 & 30.59 & - & - & 35.86 & 35.78 & 34.97 & - & - \\
\hline $302-\mathrm{N} 150$ & 1.99 & 4.93 & 4.75 & 3.96 & - & - & 6.85 & 6.61 & 5.46 & - & - \\
\hline $302-\mathrm{N} 150$ & 4.00 & 21.82 & 20.57 & 16.65 & - & - & 29.14 & 28.64 & 24.20 & - & - \\
\hline $302-\mathrm{N} 150$ & 6.00 & 32.16 & 32.08 & 31.25 & - & - & 35.66 & 35.59 & 35.22 & - & - \\
\hline
\end{tabular}


b: $a / h$ for offset circular web opening case

\begin{tabular}{|c|c|c|c|c|c|c|c|c|c|}
\hline \multirow[t]{2}{*}{ Specimen } & \multirow{2}{*}{$\begin{array}{c}\text { Thickness } \\
t \\
(\mathrm{~mm})\end{array}$} & \multicolumn{4}{|c|}{ Unfastened FEA load per web, $\mathrm{P}_{\mathrm{FEA}}$} & \multicolumn{4}{|c|}{ Fastened FEA load per web, $\mathrm{P}_{\mathrm{FEA}}$} \\
\hline & & $\begin{array}{l}A(0) \\
(k N)\end{array}$ & $\begin{array}{c}A(0.2) \\
(k N)\end{array}$ & $\begin{array}{c}A(0.4) \\
(k N)\end{array}$ & $\begin{array}{c}A(0.6) \\
(k N)\end{array}$ & $\begin{array}{l}A(0) \\
(k N)\end{array}$ & $\begin{array}{c}A(0.2) \\
(k N)\end{array}$ & $\begin{array}{c}A(0.4) \\
(k N)\end{array}$ & $\begin{array}{r}A(0.6) \\
(k N)\end{array}$ \\
\hline $142-\mathrm{N} 100$ & 1.27 & 2.23 & 2.22 & 2.18 & 2.12 & 3.11 & 3.10 & 3.05 & 2.98 \\
\hline $142-\mathrm{N} 100$ & 4.00 & 18.33 & 18.31 & 18.20 & 16.73 & 23.29 & 23.27 & 23.16 & 20.53 \\
\hline $142-\mathrm{N} 100$ & 6.00 & 28.32 & 28.28 & 28.07 & 25.53 & 33.31 & 33.26 & 32.97 & 30.04 \\
\hline $142-\mathrm{N} 120$ & 1.27 & 2.47 & 2.46 & 2.44 & 2.38 & 3.23 & 3.22 & 3.17 & 3.11 \\
\hline $142-\mathrm{N} 120$ & 4.00 & 18.06 & 18.03 & 17.92 & 16.28 & 22.96 & 22.94 & 22.82 & 20.02 \\
\hline $142-\mathrm{N} 120$ & 6.00 & 27.78 & 27.75 & 27.51 & 24.72 & 32.80 & 32.75 & 32.45 & 29.43 \\
\hline $142-\mathrm{N} 150$ & 1.28 & 2.63 & 2.63 & 2.62 & 2.54 & 3.55 & 3.55 & 3.51 & 3.44 \\
\hline $142-\mathrm{N} 150$ & 4.00 & 17.39 & 17.37 & 17.25 & 15.42 & 22.32 & 22.31 & 22.15 & 19.14 \\
\hline $142-\mathrm{N} 150$ & 6.00 & 26.92 & 26.88 & 26.60 & 23.56 & 32.11 & 32.06 & 31.71 & 28.53 \\
\hline $202-\mathrm{N} 100$ & 1.39 & 2.29 & 2.26 & 2.15 & 2.02 & 3.27 & 3.25 & 3.18 & 3.03 \\
\hline $202-\mathrm{N} 100$ & 4.00 & 19.68 & 19.60 & 19.33 & 18.44 & 27.05 & 27.00 & 26.79 & 24.54 \\
\hline $202-\mathrm{N} 100$ & 6.00 & 31.10 & 31.03 & 30.74 & 29.20 & 35.32 & 35.23 & 34.86 & 33.21 \\
\hline $202-\mathrm{N} 120$ & 1.39 & 2.43 & 2.40 & 2.29 & 2.16 & 3.52 & 3.49 & 3.41 & 3.31 \\
\hline $202-\mathrm{N} 120$ & 4.00 & 20.15 & 20.07 & 19.82 & 18.82 & 27.22 & 27.18 & 24.60 & 24.37 \\
\hline $202-\mathrm{N} 120$ & 6.00 & 30.92 & 30.85 & 30.55 & 28.88 & 35.11 & 35.03 & 33.48 & 32.96 \\
\hline $202-\mathrm{N} 150$ & 1.39 & 2.57 & 2.54 & 2.45 & 2.32 & 3.89 & 3.87 & 3.79 & 3.70 \\
\hline $202-\mathrm{N} 150$ & 4.00 & 20.03 & 19.97 & 19.73 & 18.47 & 26.74 & 26.70 & 26.47 & 23.60 \\
\hline $202-\mathrm{N} 150$ & 6.00 & 30.29 & 30.22 & 29.91 & 28.02 & 34.66 & 34.58 & 34.21 & 32.39 \\
\hline $302-\mathrm{N} 100$ & 1.98 & 4.40 & 4.39 & 4.21 & 3.95 & 5.80 & 5.72 & 5.55 & 5.36 \\
\hline $302-\mathrm{N} 100$ & 2.00 & 19.97 & 19.79 & 19.32 & 18.62 & 26.13 & 26.06 & 25.88 & 25.60 \\
\hline $302-\mathrm{N} 100$ & 4.00 & 32.38 & 32.27 & 31.82 & 30.54 & 35.96 & 35.86 & 35.43 & 34.14 \\
\hline $302-\mathrm{N} 120$ & 1.98 & 4.61 & 4.57 & 4.42 & 4.17 & 6.21 & 6.15 & 6.01 & 5.89 \\
\hline $302-\mathrm{N} 120$ & 2.00 & 20.81 & 20.64 & 20.22 & 19.51 & 28.17 & 28.10 & 27.84 & 27.06 \\
\hline $302-\mathrm{N} 120$ & 4.00 & 32.38 & 32.27 & 31.82 & 30.50 & 35.86 & 35.76 & 35.33 & 34.02 \\
\hline $302-\mathrm{N} 150$ & 1.99 & 4.93 & 4.90 & 4.75 & 4.49 & 6.85 & 6.81 & 6.70 & 6.49 \\
\hline $302-\mathrm{N} 150$ & 2.00 & 21.82 & 21.67 & 21.25 & 20.41 & 29.14 & 29.07 & 28.74 & 27.60 \\
\hline $302-\mathrm{N} 150$ & 4.00 & 32.16 & 32.06 & 31.61 & 30.29 & 35.66 & 35.56 & 35.14 & 33.84 \\
\hline
\end{tabular}


c: $x / h$ for offset circular web opening case

\begin{tabular}{|c|c|c|c|c|c|c|c|c|c|}
\hline \multirow[t]{2}{*}{ Specimen } & \multirow{2}{*}{$\begin{array}{c}\text { Thickness } \\
t \\
(\mathrm{~mm}) \\
\end{array}$} & \multicolumn{4}{|c|}{ Unfastened FEA load per web, $P(F E A)$} & \multicolumn{4}{|c|}{ Fastened FEA load per web, $\mathrm{P}_{\mathrm{FEA}}$} \\
\hline & & $\begin{array}{l}X(0) \\
(k N)\end{array}$ & $\begin{array}{c}X(0.2) \\
(k N)\end{array}$ & $\begin{array}{c}X(0.4) \\
(k N)\end{array}$ & $\begin{array}{c}X(0.6) \\
(k N)\end{array}$ & $\begin{array}{l}X(0) \\
(k N)\end{array}$ & $\begin{array}{c}X(0.2) \\
(k N)\end{array}$ & $\begin{array}{c}X(0.4) \\
(k N)\end{array}$ & $\begin{array}{c}X(0.6) \\
(k N)\end{array}$ \\
\hline $142-\mathrm{N} 100-\mathrm{A} 0$ & 1.27 & 2.35 & 2.35 & 2.35 & 2.35 & 2.21 & 2.21 & 2.21 & 2.21 \\
\hline $142-\mathrm{N} 100-\mathrm{A} 0.2$ & 1.27 & 2.30 & 2.31 & 2.32 & 2.33 & 2.16 & 2.17 & 2.18 & 2.19 \\
\hline $142-\mathrm{N} 100-\mathrm{A} 0.4$ & 1.27 & 2.15 & 2.19 & 2.24 & 2.27 & 2.02 & 2.06 & 2.10 & 2.13 \\
\hline $142-\mathrm{N} 100-\mathrm{A} 0.6$ & 1.27 & 1.97 & 2.05 & 2.13 & 2.20 & 1.84 & 1.92 & 2.00 & 2.05 \\
\hline 142-N100-A0.8 & 1.27 & --- & --- & --- & --- & --- & --- & --- & --- \\
\hline 142-N120-A0 & 1.27 & 2.52 & 2.52 & 2.52 & 2.52 & 2.44 & 2.44 & 2.44 & 2.44 \\
\hline 142-N120-A0.2 & 1.27 & 2.47 & 2.48 & 2.49 & 2.50 & 2.40 & 2.40 & 2.42 & 2.42 \\
\hline 142-N120-A0.4 & 1.27 & 2.33 & 2.37 & 2.41 & 2.45 & 2.26 & 2.30 & 2.34 & 2.37 \\
\hline 142-N120-A0.6 & 1.27 & 2.17 & 2.25 & 2.32 & 2.38 & 2.10 & 2.17 & 2.24 & 2.30 \\
\hline 142-N120-A0.8 & 1.27 & --- & --- & --- & --- & --- & --- & --- & --- \\
\hline $142-\mathrm{N} 150-\mathrm{A} 0$ & 1.28 & 2.77 & 2.77 & 2.77 & 2.77 & 2.60 & 2.60 & 2.60 & 2.60 \\
\hline $142-\mathrm{N} 150-\mathrm{A} 0.2$ & 1.28 & 2.72 & 2.73 & 2.74 & 2.75 & 2.55 & 2.56 & 2.57 & 2.58 \\
\hline $142-\mathrm{N} 150-\mathrm{A} 0.4$ & 1.28 & 2.60 & 2.64 & 2.65 & 2.70 & 2.44 & 2.47 & 2.51 & 2.54 \\
\hline 142-N150-A0.6 & 1.28 & 2.45 & 2.52 & 2.59 & 2.64 & 2.30 & 2.36 & 2.42 & 2.47 \\
\hline 142-N150-A0.8 & 1.28 & 2.32 & 2.41 & 2.43 & 2.35 & 2.17 & 2.27 & 2.29 & 2.53 \\
\hline 202-N100-A0 & 1.39 & 2.25 & 2.25 & 2.25 & 2.25 & 2.25 & 2.25 & 2.25 & 2.25 \\
\hline 202-N100-A0.2 & 1.39 & 2.20 & 2.20 & 2.21 & 2.22 & 2.20 & 2.20 & 2.21 & 2.22 \\
\hline 202-N100-A0.4 & 1.39 & 2.03 & 2.06 & 2.09 & 2.11 & 2.03 & 2.06 & 2.09 & 2.11 \\
\hline 202-N100-A0.6 & 1.39 & 1.78 & 1.85 & 1.92 & 1.98 & 2.37 & 2.37 & 2.37 & 2.37 \\
\hline 202-N120-A0 & 1.39 & 2.32 & 2.32 & 2.32 & 2.32 & 2.32 & 2.33 & 2.33 & 2.34 \\
\hline 202-N120-A0.2 & 1.39 & 2.27 & 2.28 & 2.28 & 2.29 & 2.16 & 2.19 & 2.21 & 2.24 \\
\hline 202-N120-A0.4 & 1.39 & 2.16 & 2.19 & 2.21 & 2.24 & 1.92 & 1.99 & 2.06 & 2.11 \\
\hline 202-N120-A0.6 & 1.39 & 1.92 & 1.99 & 2.06 & 2.11 & --- & --- & --- & --- \\
\hline 202-N150-A0 & 1.45 & 2.51 & 2.51 & 2.51 & 2.51 & 2.51 & 2.51 & 2.51 & 2.51 \\
\hline 202-N150-A0.2 & 1.45 & 2.45 & 2.46 & 2.47 & 2.47 & 2.45 & 2.46 & 2.47 & 2.47 \\
\hline 202-N150-A0.4 & 1.45 & 2.30 & 2.33 & 2.35 & 2.38 & 2.30 & 2.33 & 2.35 & 2.38 \\
\hline 202-N150-A0.6 & 1.45 & 2.10 & 2.17 & 2.23 & 2.26 & 2.10 & 2.17 & 2.23 & 2.26 \\
\hline $302-\mathrm{N} 100-\mathrm{A} 0$ & 1.98 & 4.05 & 4.05 & 4.05 & 4.05 & 4.37 & 4.37 & 4.37 & 4.37 \\
\hline 302-N100-A0.2 & 1.98 & 3.95 & 3.97 & 4.01 & 4.05 & 4.26 & 4.30 & 4.34 & 4.34 \\
\hline 302-N120-A0 & 1.96 & 4.21 & 4.21 & 4.21 & 4.21 & 4.57 & 4.57 & 4.57 & 4.57 \\
\hline 302-N120-A0.2 & 1.96 & 4.14 & 4.18 & 4.22 & 4.23 & 4.46 & 4.50 & 4.54 & 4.54 \\
\hline 302-N120-A0.4 & 1.96 & 3.83 & 3.97 & 4.04 & 4.05 & 4.38 & 4.25 & 4.36 & 4.46 \\
\hline 302-N120-A0.6 & 1.96 & 3.38 & 3.61 & 3.72 & 3.78 & --- & --- & --- & --- \\
\hline $302-\mathrm{N} 150-\mathrm{A} 0$ & 1.99 & 4.53 & 4.53 & 4.53 & 4.53 & 4.87 & 4.87 & 4.87 & 4.87 \\
\hline 302-N150-A0.2 & 1.99 & 4.43 & 4.48 & 4.51 & 4.50 & 4.76 & 4.81 & 4.85 & 4.84 \\
\hline 302-N150-A0.4 & 1.99 & 4.11 & 4.24 & 4.31 & 4.32 & 4.43 & 4.57 & 4.66 & 4.68 \\
\hline $302-\mathrm{N} 150-\mathrm{A} 0.6$ & 1.99 & 3.68 & 3.89 & 3.99 & 4.06 & --- & --- & --- & --- \\
\hline
\end{tabular}


Table 6: Comparison of web crippling strength reduction factor for cold-formed stainless steel lipped channel-sections with reduction factors equations proposed by Lian et al. $(2016 \mathrm{c}, \mathrm{d})$

a: Flange unfastened to the bearing plate

\begin{tabular}{|c|c|c|c|c|c|c|c|c|c|c|c|c|c|c|}
\hline \multirow{3}{*}{ Specimen } & \multirow{3}{*}{$\begin{array}{l}\text { Factored resistance } \\
\text { (Eqn. 1) }\end{array}$} & \multirow{3}{*}{$\begin{array}{c}\text { Factored resistance } \\
\text { (Eqn. 3) }\end{array}$} & \multicolumn{5}{|c|}{ Reduction factor } & \multicolumn{7}{|c|}{ Comparison with resistance from Lian } \\
\hline & & & \multirow{2}{*}{\multicolumn{2}{|c|}{$\begin{array}{c}\text { Duplex } \\
R=P(\text { Opening }) / P(A 0)\end{array}$}} & \multirow{2}{*}{\multicolumn{2}{|c|}{$\begin{array}{c}\text { Ferritic } \\
R=P(\text { Opening }) / P(A 0)\end{array}$}} & \multirow{2}{*}{\multicolumn{2}{|c|}{$\begin{array}{c}\text { Austenitic } \\
R=P(\text { Opening }) / P(A 0)\end{array}$}} & \multirow{2}{*}{\multicolumn{2}{|c|}{ Duplex }} & \multirow[t]{2}{*}{ Ferritic } & \multirow{2}{*}{\multicolumn{3}{|c|}{ Austenitic }} \\
\hline & & & & & & & & & & & & & & \\
\hline & Centred & Offset & Centred & Offset & Centred & Offset & Centred & Offset & Centred & Offset & Centred & Offset & Centred & Offset \\
\hline 142-N100-MA0.6-FR & 0.82 & 0.90 & 0.68 & 0.92 & 0.67 & 0.93 & 0.68 & 0.95 & 0.82 & 1.03 & 0.82 & 1.03 & 0.83 & 1.05 \\
\hline 142-N120-MA0.6-FR & 0.82 & 0.90 & 0.70 & 0.93 & 0.70 & 0.94 & 0.71 & 0.96 & 0.86 & 1.04 & 0.85 & 1.05 & 0.86 & 1.06 \\
\hline 142-N150-MA0.4-FR & 0.89 & 0.95 & 0.85 & 0.96 & 0.73 & 0.97 & 1.15 & 0.97 & 0.96 & 1.01 & 0.82 & 1.01 & 1.29 & 1.02 \\
\hline 202-N100-MA0.4-FR & 0.87 & 0.95 & 0.84 & 0.93 & 0.83 & 0.93 & 0.83 & 0.94 & 0.96 & 0.98 & 0.95 & 0.98 & 0.95 & 0.99 \\
\hline 202-N100-MA0.6-FR & 0.94 & 1.00 & 0.97 & 0.98 & 0.97 & 0.98 & 0.98 & 0.98 & 1.04 & 0.98 & 1.04 & 0.98 & 1.05 & 0.98 \\
\hline 202-N120-MA0.4-FR & 0.87 & 0.95 & 0.86 & 0.93 & 0.84 & 0.93 & 0.85 & 0.95 & 0.98 & 0.98 & 0.96 & 0.98 & 0.97 & 1.00 \\
\hline 202-N120-MA0.6-FR & 0.80 & 0.90 & 0.67 & 0.88 & 0.66 & 0.89 & 0.66 & 0.92 & 0.84 & 0.98 & 0.83 & 0.99 & 0.83 & 1.03 \\
\hline 202-N150-MA0.4-FR & 0.87 & 0.95 & 0.87 & 0.95 & 0.85 & 0.94 & 0.86 & 0.96 & 1.00 & 0.99 & 0.98 & 0.99 & 0.98 & 1.01 \\
\hline 302-N100-MA0.6-FR & 0.92 & 1.00 & 0.97 & 0.98 & 0.97 & 0.98 & 0.97 & 0.99 & 1.05 & 0.98 & 1.05 & 0.98 & 1.06 & 0.99 \\
\hline 302-N120-MA0.6-FR & 0.85 & 0.95 & 0.80 & 0.95 & 0.79 & 0.95 & 0.79 & 0.95 & 0.94 & 1.00 & 0.92 & 1.00 & 0.92 & 1.00 \\
\hline 302-N150-MA0.6-FR & 0.92 & 1.00 & 0.97 & 0.99 & 0.96 & 0.99 & 0.80 & 0.99 & 1.06 & 0.98 & 1.04 & 0.99 & 0.87 & 0.99 \\
\hline Mean, Pm & & & & & & & & & 0.95 & 0.99 & 0.93 & 1.00 & 0.96 & 1.01 \\
\hline \multicolumn{15}{|l|}{ Coefficient of } \\
\hline variation, $\mathrm{Vp}$ & & & & & & & & & 0.09 & 0.02 & 0.10 & 0.02 & 0.14 & 0.03 \\
\hline
\end{tabular}




\section{b: Flange fastened to the bearing plate}

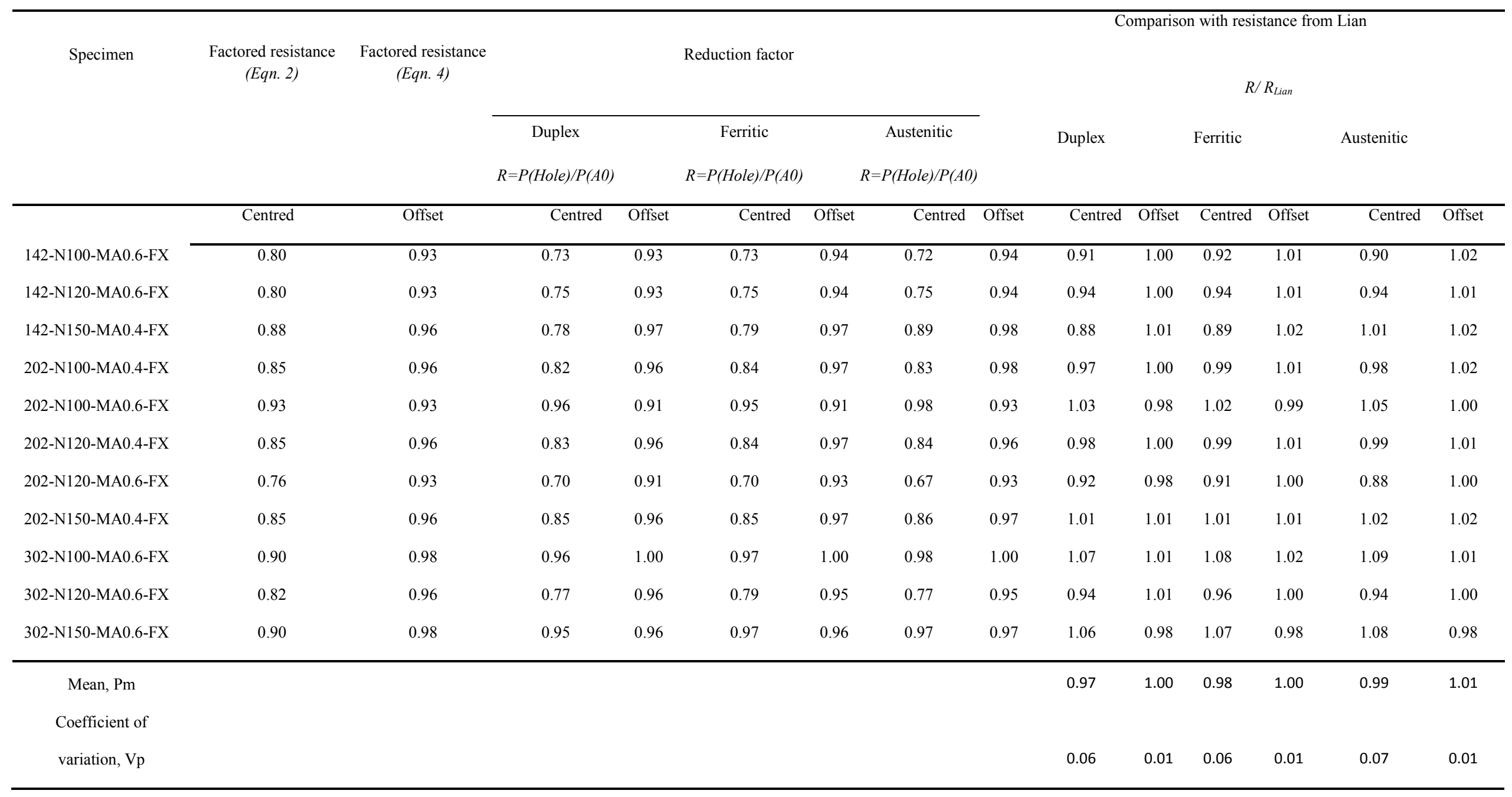


Table 7: Coefficients of the proposed strength reduction factor equations

Strength reduction factor (Eqns. 5 and 6)

\begin{tabular}{|c|c|c|c|}
\hline Stainless steel grade & Coefficient & $\begin{array}{l}\text { Unfastened to } \\
\text { bearing plate }\end{array}$ & $\begin{array}{l}\text { Fastened to } \\
\text { bearing plate }\end{array}$ \\
\hline \multirow{6}{*}{ EN 1.4462 (Duplex) } & $\alpha$ & 0.98 & 0.98 \\
\hline & $\gamma$ & 0.43 & 0.35 \\
\hline & $\lambda$ & 0.14 & 0.11 \\
\hline & $\rho$ & 0.96 & 0.98 \\
\hline & $\mu$ & 0.12 & 0.07 \\
\hline & $\zeta$ & 0.06 & 0.06 \\
\hline \multirow{6}{*}{$\begin{array}{l}\text { EN } 1.4404 \\
\text { (Austenitic) }\end{array}$} & $\alpha$ & 0.95 & 0.98 \\
\hline & $\gamma$ & 0.46 & 0.40 \\
\hline & $\lambda$ & 0.18 & 0.13 \\
\hline & $\rho$ & 0.96 & 0.98 \\
\hline & $\mu$ & 0.14 & 0.06 \\
\hline & $\zeta$ & 0.06 & 0.04 \\
\hline \multirow{6}{*}{ EN 1.4003 (Ferritic) } & $\alpha$ & 0.97 & 0.97 \\
\hline & $\gamma$ & 0.45 & 0.39 \\
\hline & $\lambda$ & 0.16 & 0.13 \\
\hline & $\rho$ & 0.95 & 0.97 \\
\hline & $\mu$ & 0.13 & 0.08 \\
\hline & $\zeta$ & 0.07 & 0.04 \\
\hline
\end{tabular}


Table 8: Statistical analysis of strength reduction factor

a: Duplex stainless steel grade

\begin{tabular}{ccccc}
\hline \multirow{2}{*}{ Statistical parameters } & \multicolumn{2}{c}{$\begin{array}{c}\text { Centred circular web opening } \\
R_{(F E A)} / R_{p}\end{array}$} & \multicolumn{2}{c}{$\begin{array}{c}\text { Offset circular web opening } \\
R_{(F E A)} / R_{p}\end{array}$} \\
\cline { 2 - 5 } & $\begin{array}{c}\text { Unfastened } \\
\text { to bearing plate }\end{array}$ & $\begin{array}{c}\text { Fastened } \\
\text { to bearing plate }\end{array}$ & $\begin{array}{c}\text { Unfastened } \\
\text { to bearing plate }\end{array}$ & $\begin{array}{c}\text { Fastened } \\
\text { to bearing plate }\end{array}$ \\
\hline Number of data & 69 & 69 & 84 & 81 \\
Mean, $P_{m}$ & 0.99 & 1.00 & 1.04 & 1.04 \\
Coefficient of variation, $V_{p}$ & 0.09 & 0.08 & 0.04 & 0.05 \\
Reliability index, $\beta$ & 2.62 & 2.69 & 2.97 & 2.95 \\
Resistance factor, $\phi$ & 0.85 & 0.85 & 0.85 & 0.85 \\
\hline
\end{tabular}


b: Austenitic stainless steel grade

\begin{tabular}{ccccc}
\hline \multirow{2}{*}{ Statistical parameters } & \multicolumn{2}{c}{$\begin{array}{c}\text { Centred circular web opening } \\
R_{(F E A)} / R_{p}\end{array}$} & \multicolumn{2}{c}{$\begin{array}{c}\text { Offset circular web opening } \\
R_{(F E A)} / R_{p}\end{array}$} \\
\cline { 2 - 5 } & $\begin{array}{c}\text { Unfastened } \\
\text { to bearing plate }\end{array}$ & $\begin{array}{c}\text { Fastened } \\
\text { to bearing plate }\end{array}$ & $\begin{array}{c}\text { Unfastened } \\
\text { to bearing plate }\end{array}$ & $\begin{array}{c}\text { Fastened } \\
\text { to bearing plate }\end{array}$ \\
\hline Number of data & 69 & 69 & 66 & 69 \\
Mean, $P_{m}$ & 1.00 & 1.00 & 1.00 & 1.00 \\
Coefficient of variation, $V_{p}$ & 0.09 & 0.08 & 0.01 & 0.01 \\
Reliability index, $\beta$ & 2.66 & 2.70 & 2.85 & 2.85 \\
Resistance factor, $\phi$ & 0.85 & 0.85 & 0.85 & 0.85 \\
\hline
\end{tabular}


c: Ferritic stainless steel grade

\begin{tabular}{ccccc}
\hline \multirow{2}{*}{ Statistical parameters } & \multicolumn{2}{c}{$\begin{array}{c}\text { Centred circular web opening } \\
R_{(F E A)} / R_{p}\end{array}$} & \multicolumn{2}{c}{$\begin{array}{c}\text { Offset circular web opening } \\
R_{(F E A)} / R_{p}\end{array}$} \\
\cline { 2 - 5 } & $\begin{array}{c}\text { Unfastened } \\
\text { to bearing plate }\end{array}$ & $\begin{array}{c}\text { Fastened } \\
\text { to bearing plate }\end{array}$ & $\begin{array}{c}\text { Unfastened } \\
\text { to bearing plate }\end{array}$ & $\begin{array}{c}\text { Fastened } \\
\text { to bearing plate }\end{array}$ \\
\hline Number of data & 69 & 69 & 66 & 69 \\
Mean, $P_{m}$ & 1.00 & 1.00 & 1.00 & 1.00 \\
Coefficient of variation, $V_{p}$ & 0.10 & 0.08 & 0.01 & 0.01 \\
Reliability index, $\beta$ & 2.62 & 2.70 & 2.85 & 2.85 \\
Resistance factor, $\phi$ & 0.85 & 0.85 & 0.85 & 0.85 \\
\hline
\end{tabular}




\section{Figure Captions:}

Figure 1: Photograph of cold-formed stainless steel lipped channel-sections with circular web openings after Lawson et. al. (2015)

Figure 2: End-one-flange (EOF) loading condition after Lian et al. (2016c,d)

(a) Circular web opening centred under bearing plate

(b) Circular web openings offset from bearing plate

Figure 3: Experimental analysis of cold-formed steel lipped channel-sections under endone-flange (EOF) loading condition for the case of flange unfastened to bearing plate after Lian et al. (2016c,d);

(a) Centred circular web opening

(b) Offset circular web opening

Figure 4: Definition of symbols

Figure 5: Finite element model of cold-formed steel lipped channel-sections under end-oneflange (EOF) loading condition for the case of flange unfastened to bearing plate after Lian et al. $(2016 \mathrm{c}, \mathrm{d})$;

(a) Centred circular web opening

(b) Offset circular web opening

Figure 6: Comparison of finite element analysis and experimental results with Lian et al. $(2016 \mathrm{c}, \mathrm{d})$

(a) Centred circular web opening for the case of flange unfastened to bearing plate

(b) Offset circular web opening for the case of flange fastened to bearing plate

Figure 7: Variation in reduction factors for $\mathrm{C} 142$ section for the case of flange unfastened to bearing plate

(a) $a / h$ for centered circular web opening

(b) $x / h$ for offset circular web opening

Figure 8: Comparison of strength reduction factor for $\mathrm{C} 142$ section for the case of flange unfastened to bearing plate

(a) with centered circular web opening

(b) with offset circular web opening 


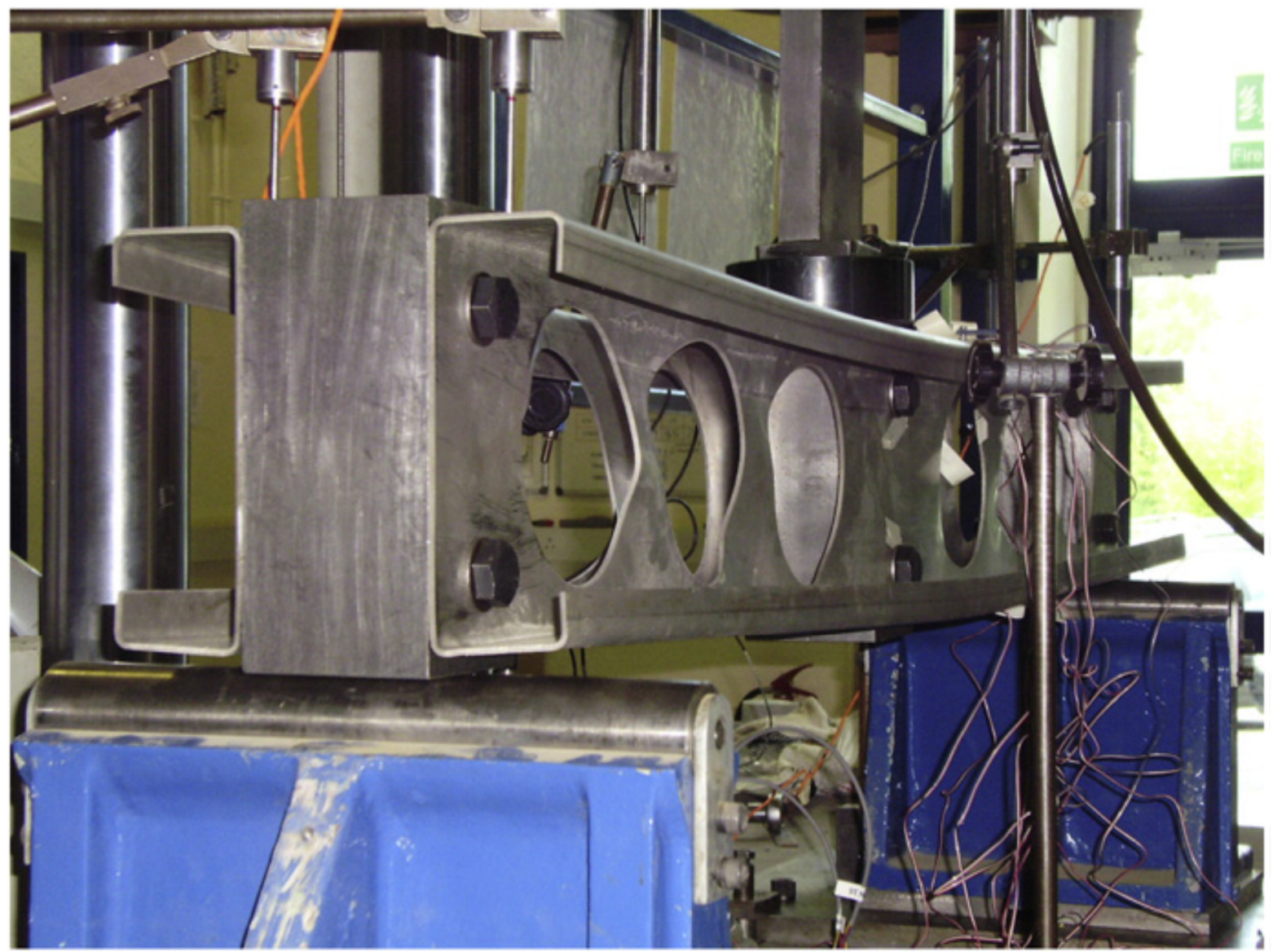

Figure 1: Photograph of cold-formed stainless steel lipped channel-sections with circular web openings after Lawson et. al. (2015) 


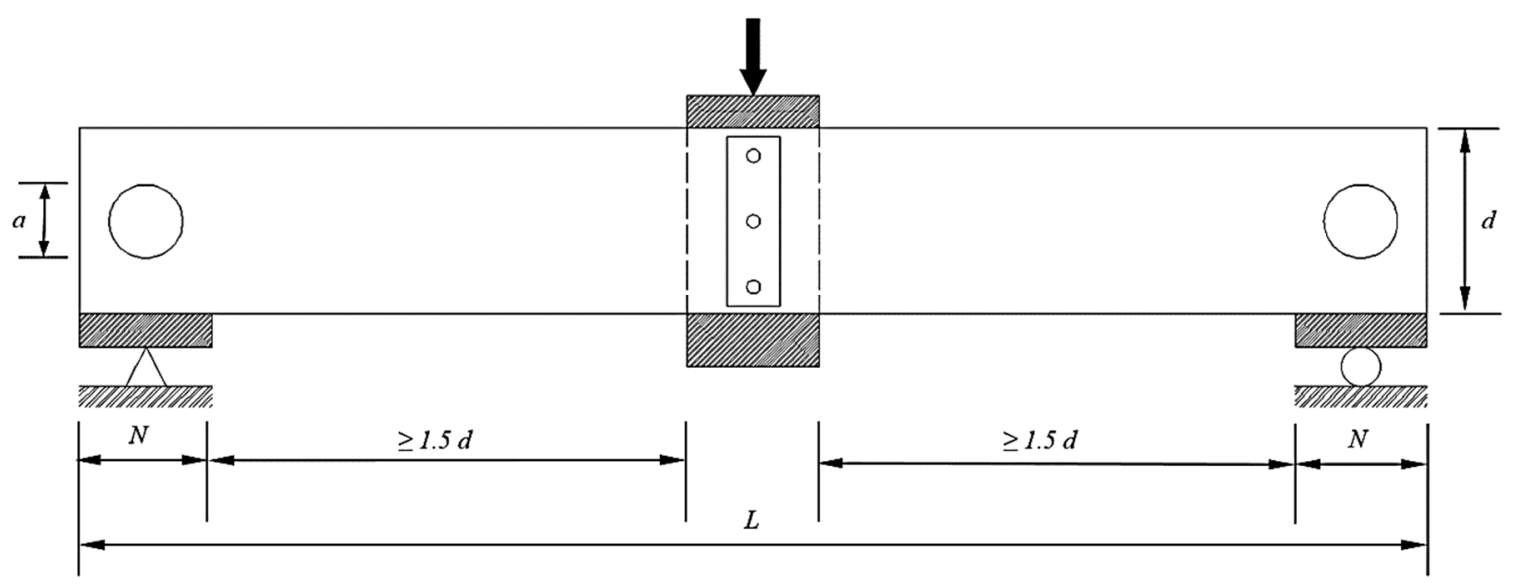

(a) Circular web opening centred under bearing plate

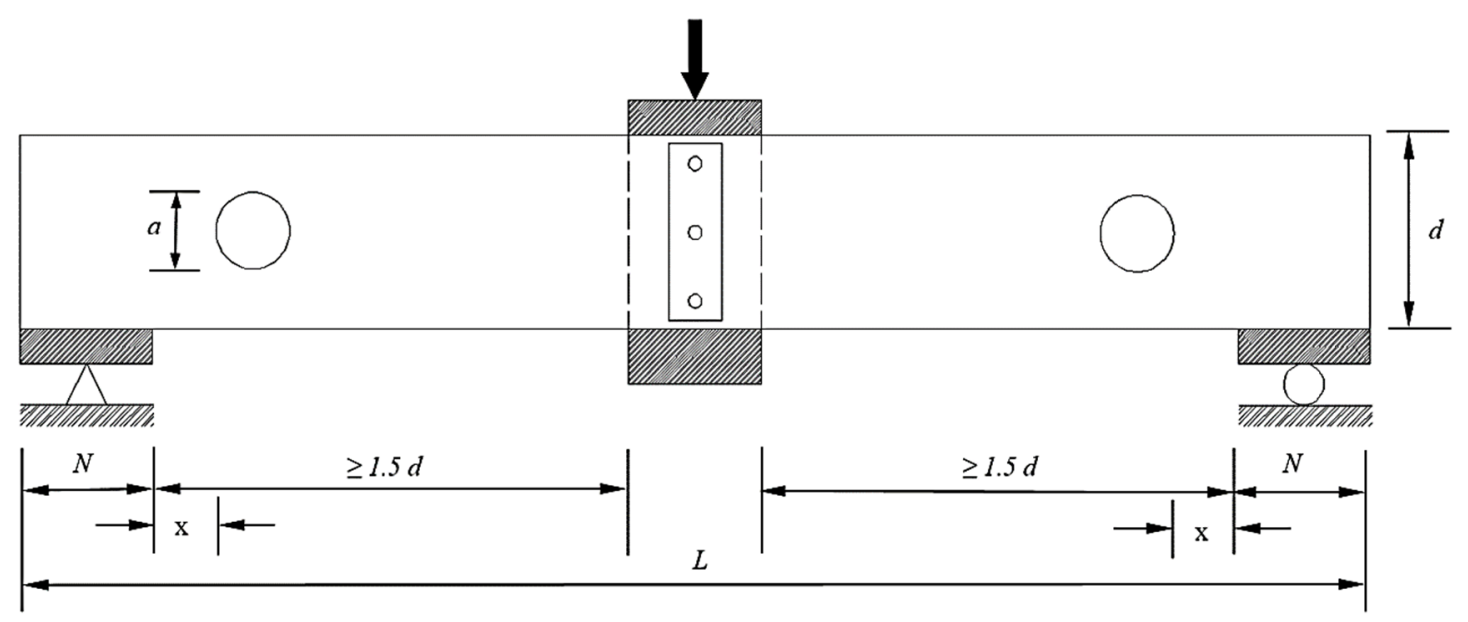

(b) Circular web openings offset from bearing plate

Figure 2: End-one-flange (EOF) loading condition after Lian et al. (2016c,d) 


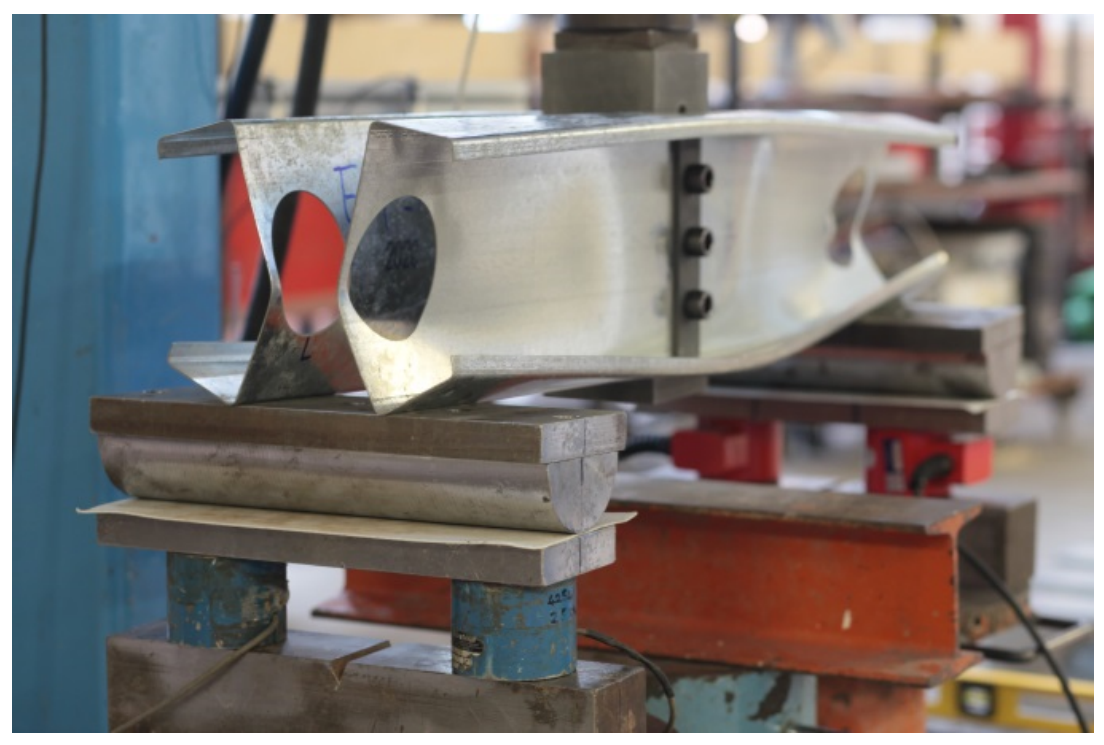

(a) Centred circular web opening

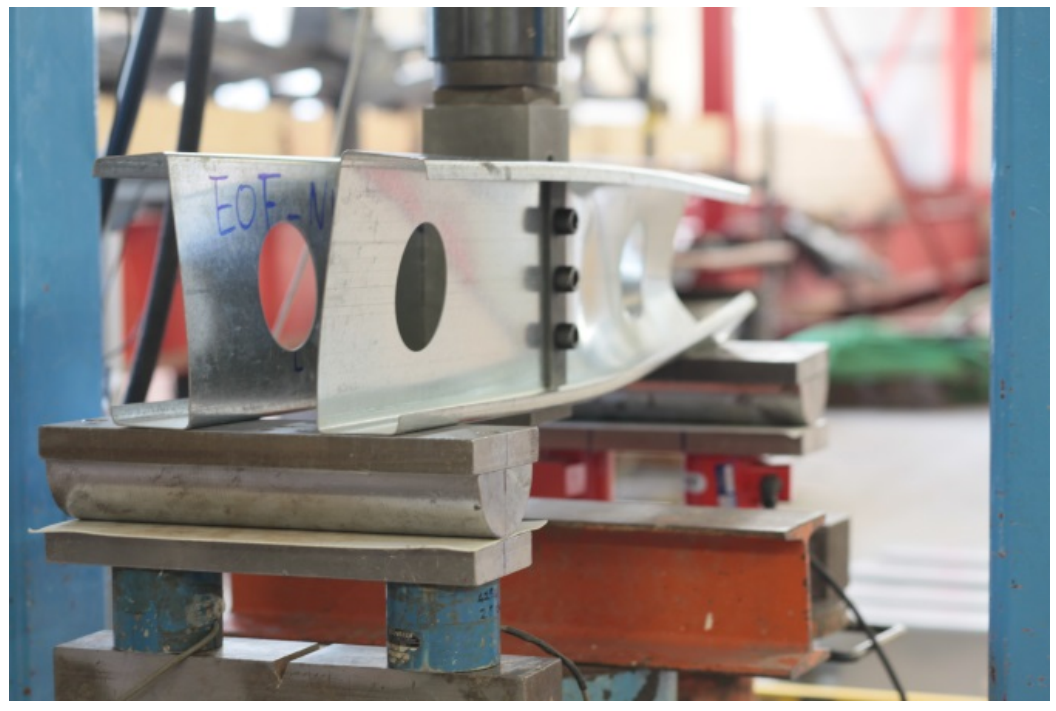

(b) Offset circular web opening

Figure 3: Experimental analysis of cold-formed steel lipped channel-sections under endone-flange (EOF) loading condition for the case of flange unfastened to bearing plate after Lian et al. $(2016 \mathrm{c}, \mathrm{d})$ 


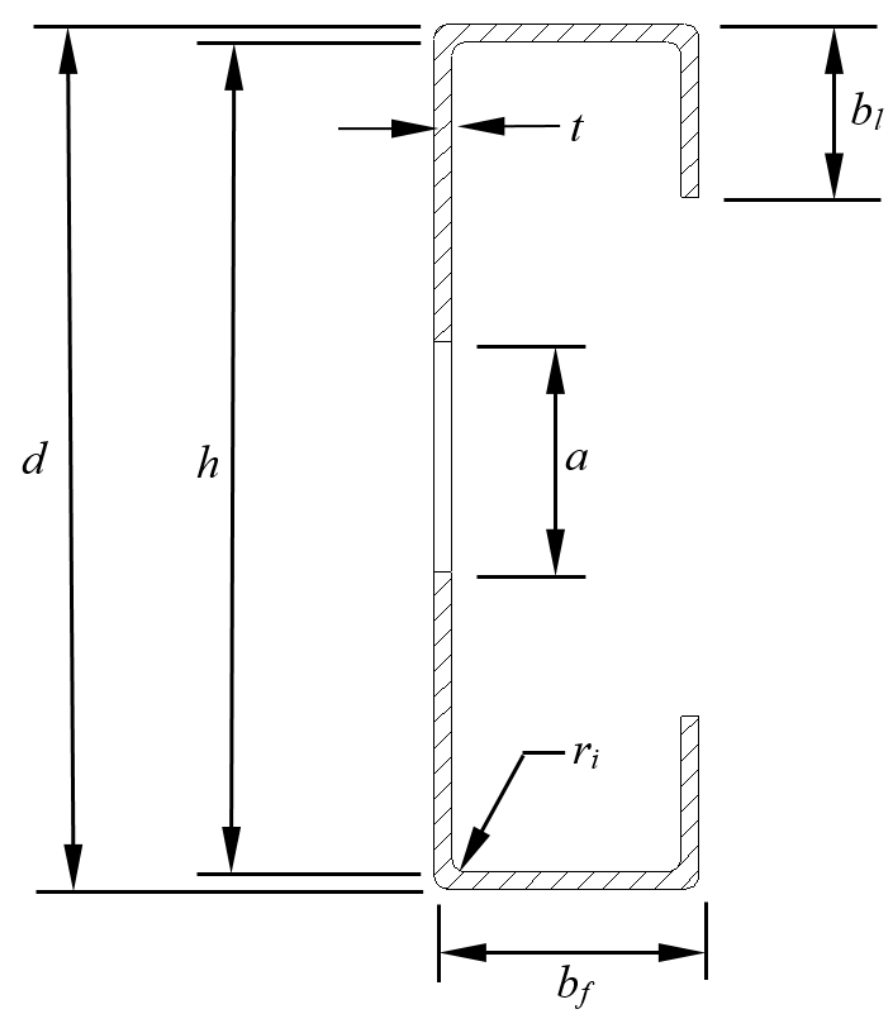

Figure 4: Definition of symbols 


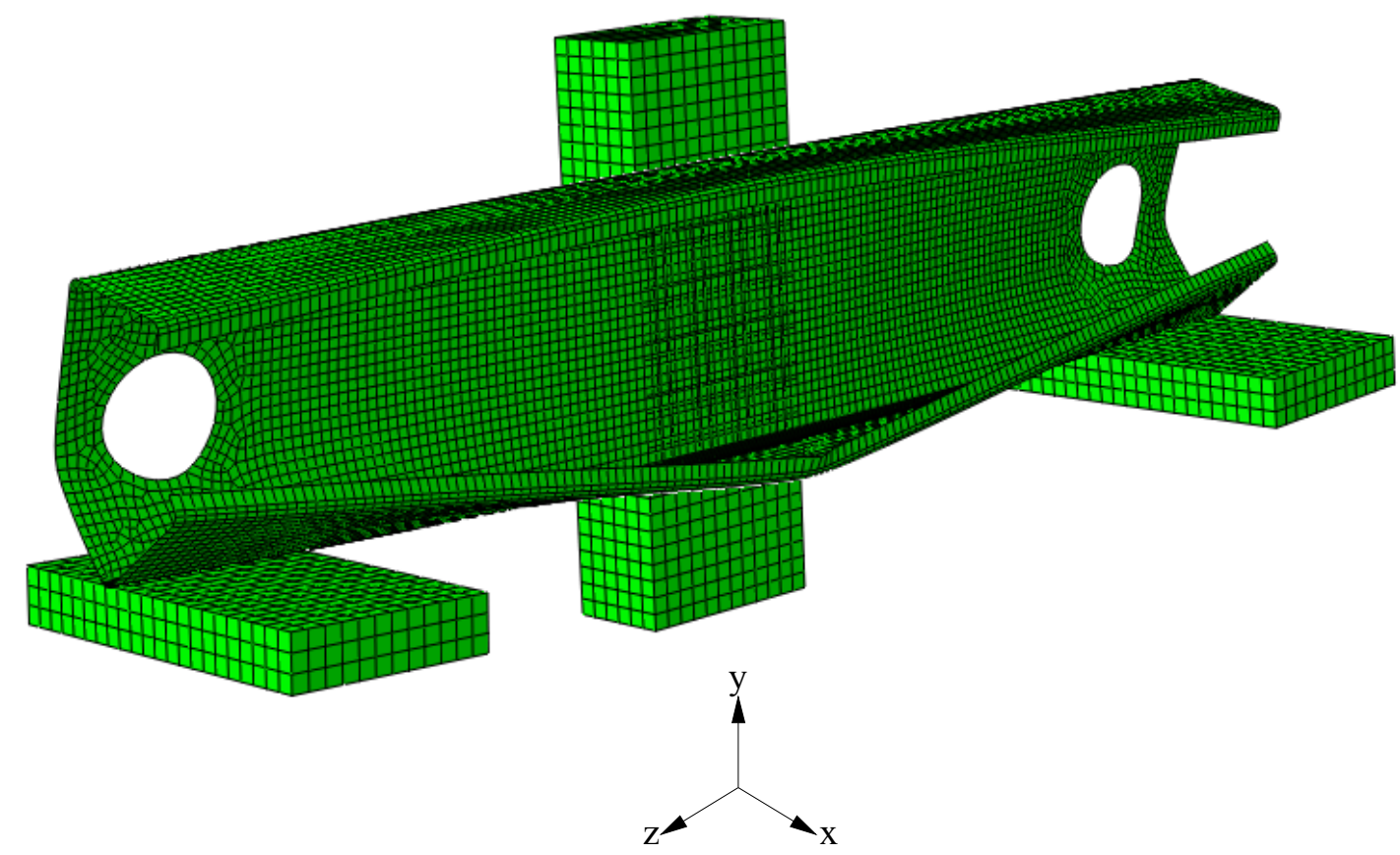

(a) Centred circular web opening

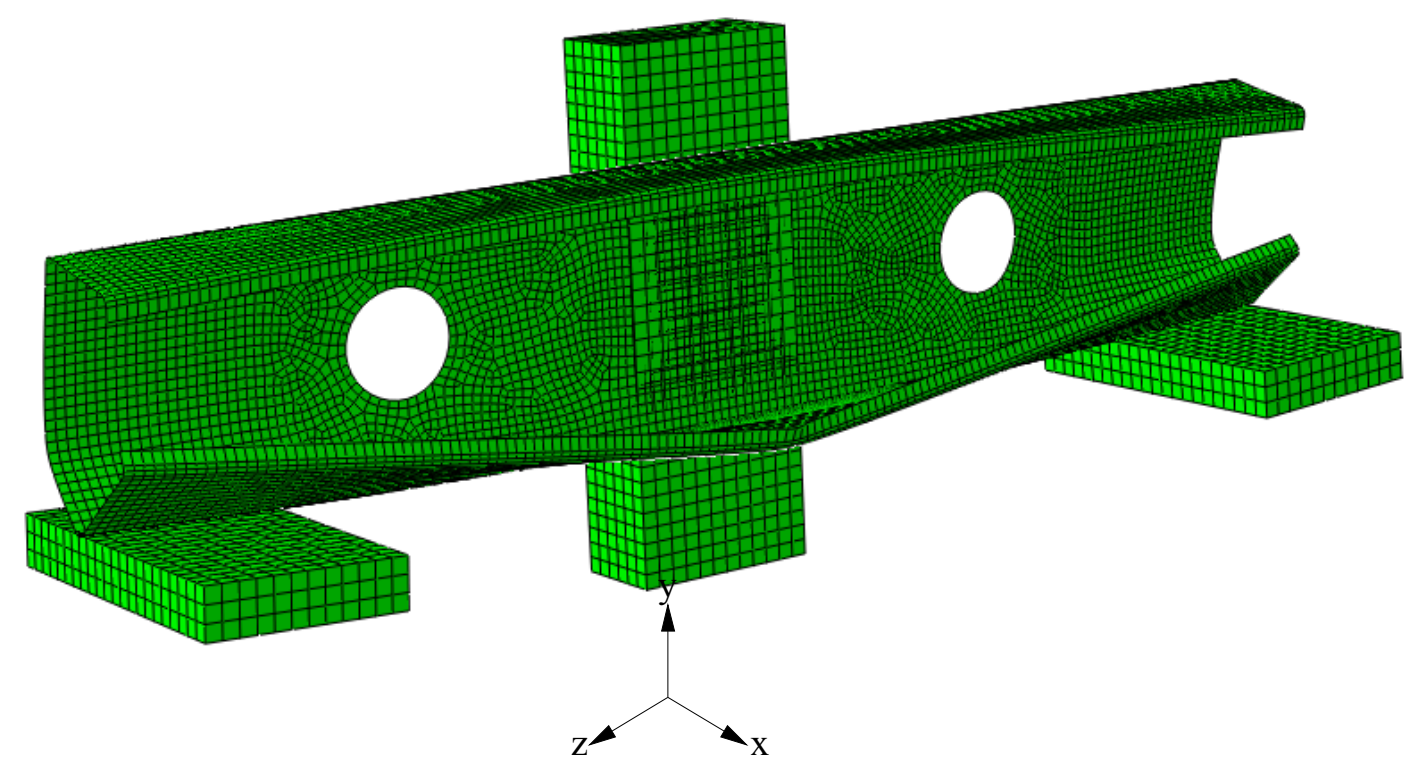

(b) Offset circular web opening

Figure 5: Deformed shape predicted from finite element analysis of cold-formed steel lipped channel-sections under end-one-flange (EOF) loading condition for case of flange unfastened to bearing plate after Lian et al. $(2016 \mathrm{c}, \mathrm{d})$ 


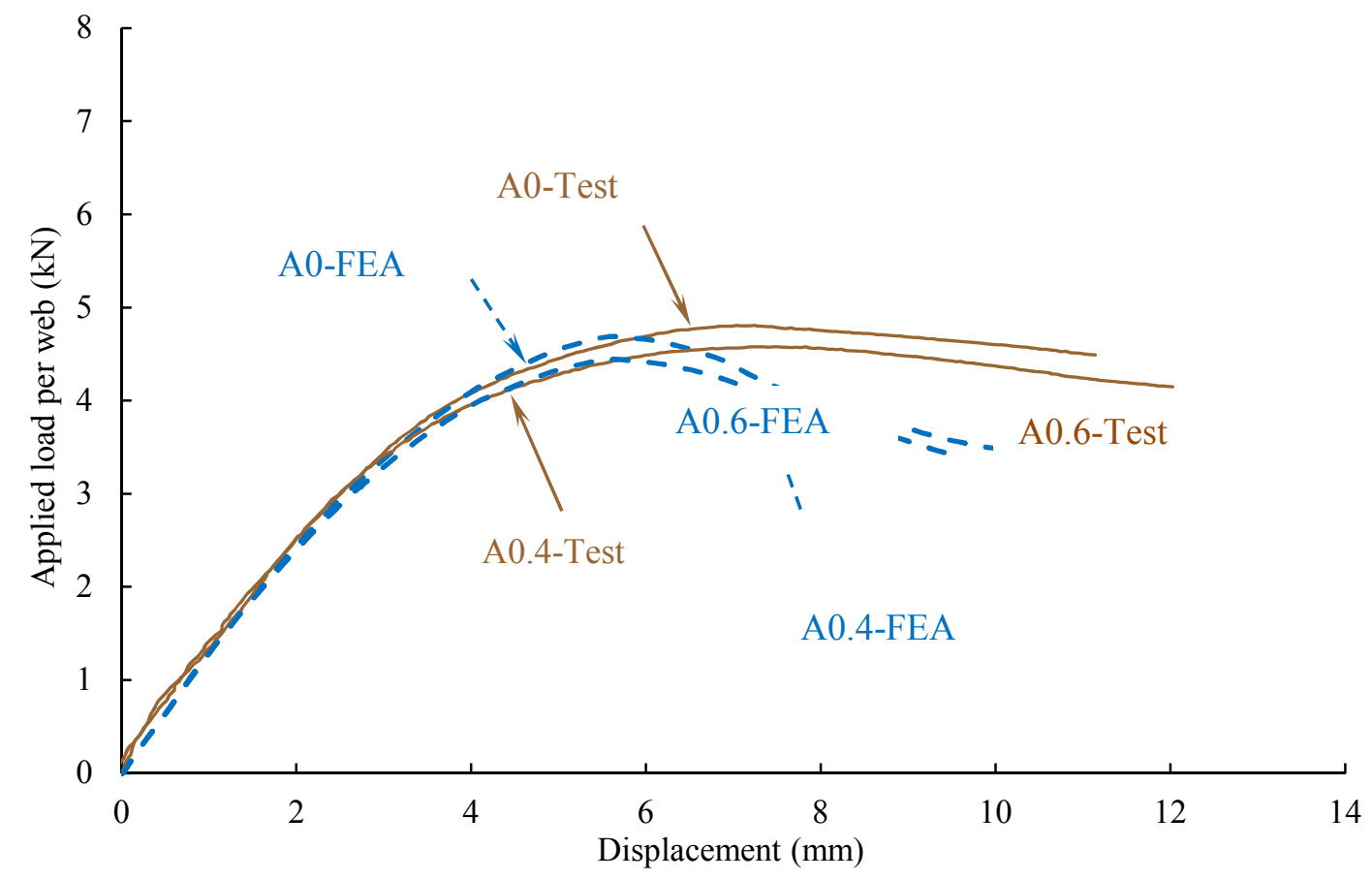

(a) Centred circular web opening for the case of flange unfastened to bearing plate 


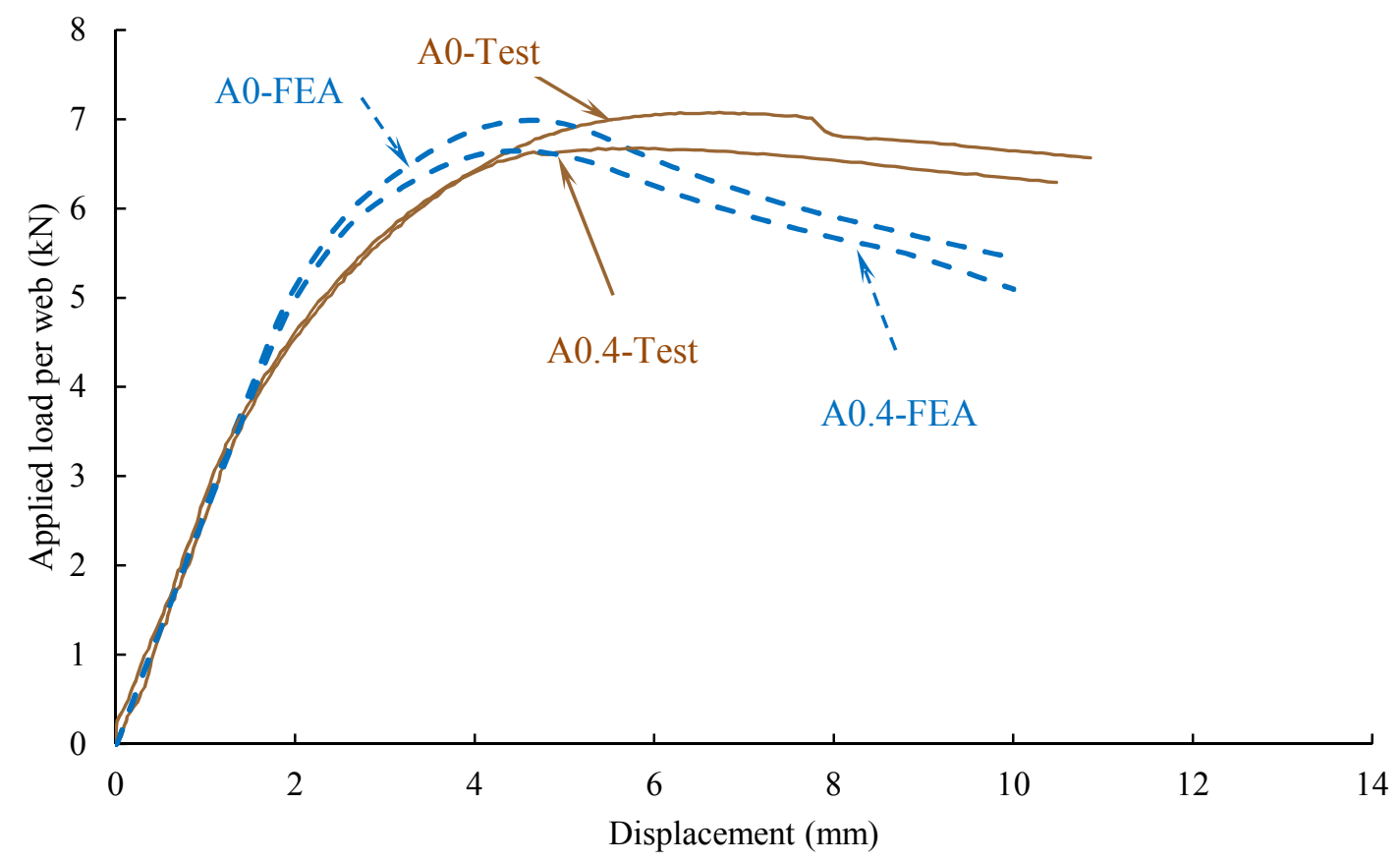

(b) Offset circular web opening for the case of flange fastened to bearing plate

Figure 6: Comparison of finite element results and experimental test results (Lian et al. 2016c,d) 


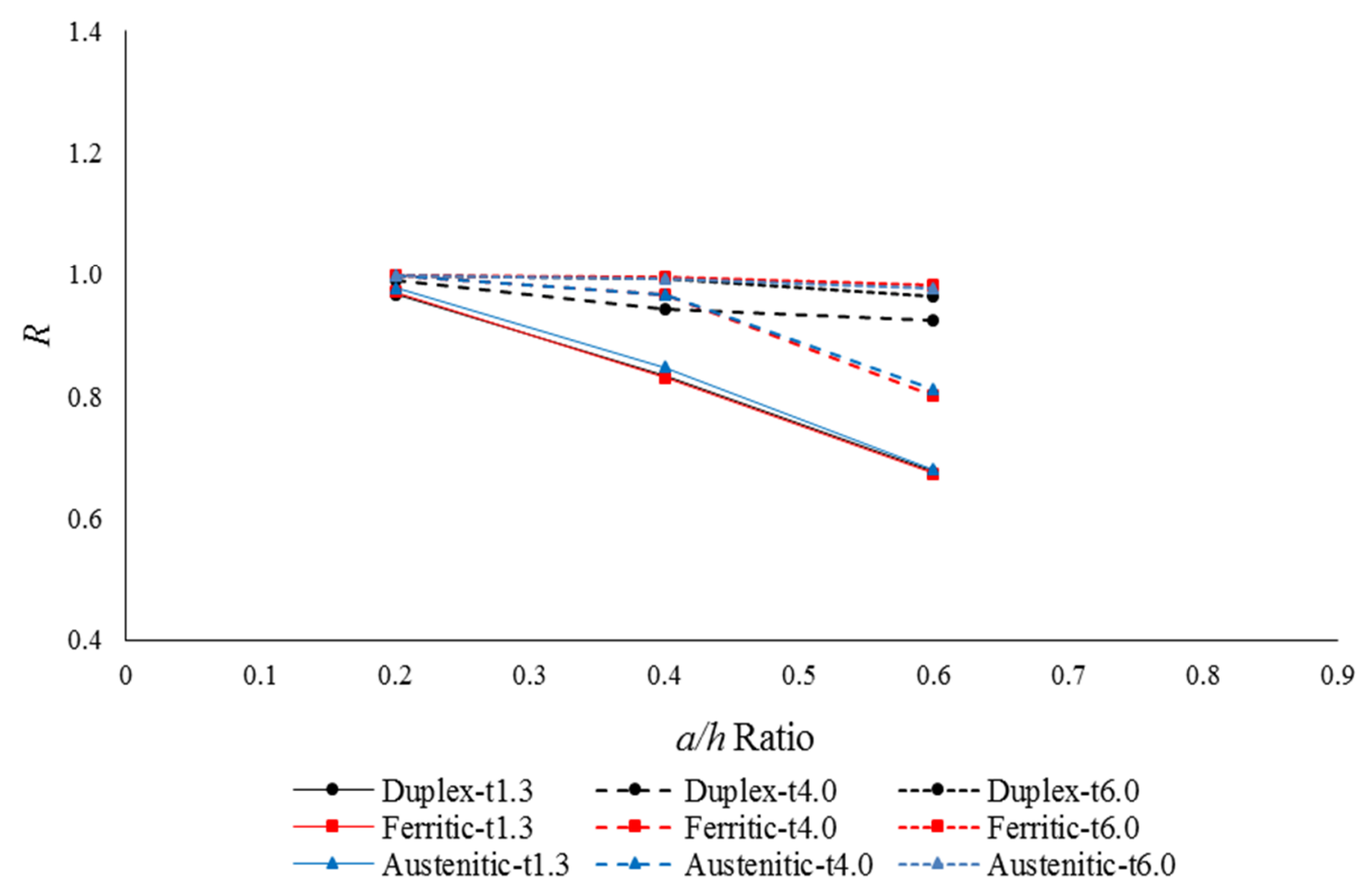

(a) $a / h$ for centered circular web opening

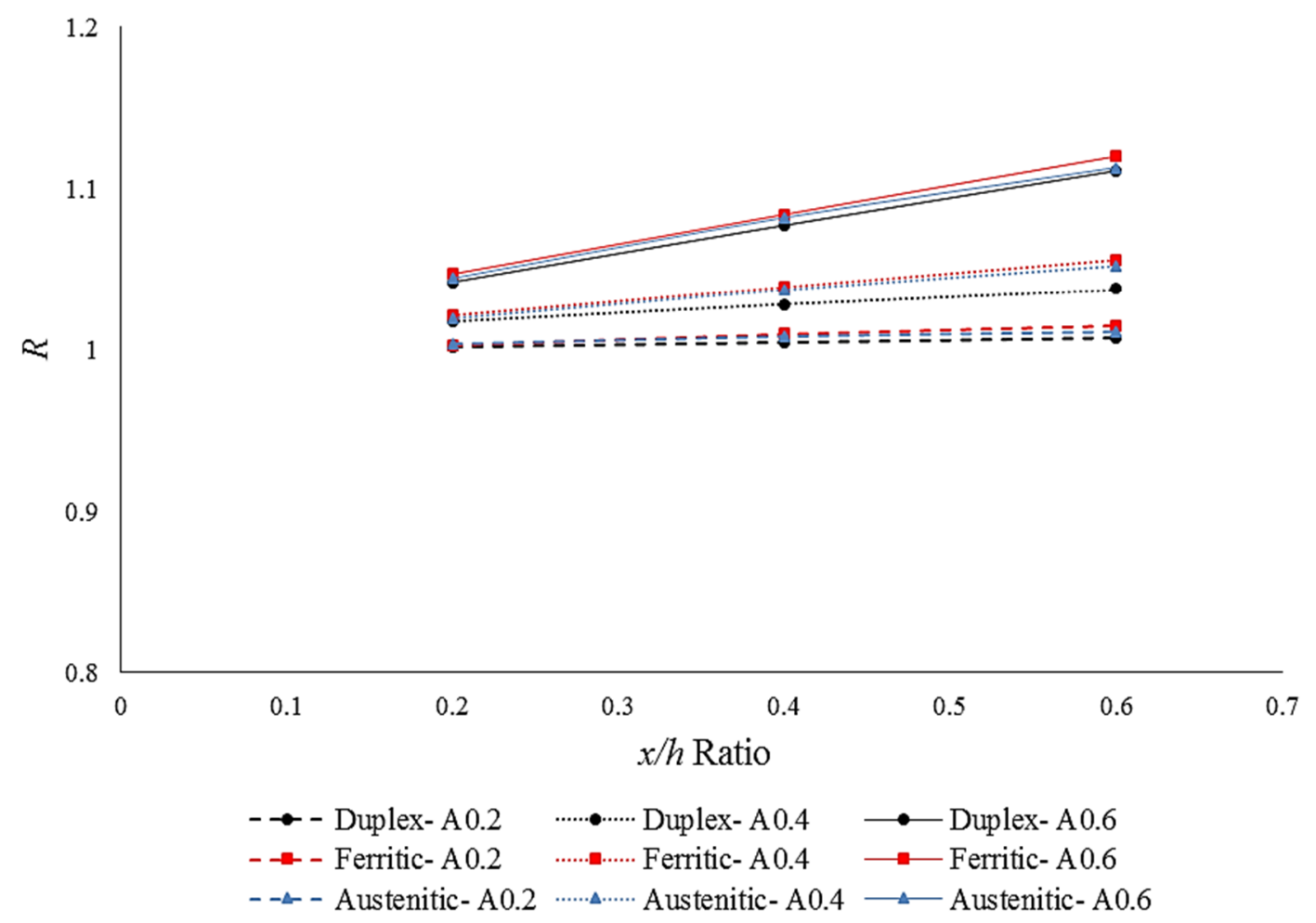

(b) $x / h$ for offset circular web opening

Figure 7: Variation in reduction factors for $\mathrm{C} 142$ section for the case of flange unfastened to bearing plate 


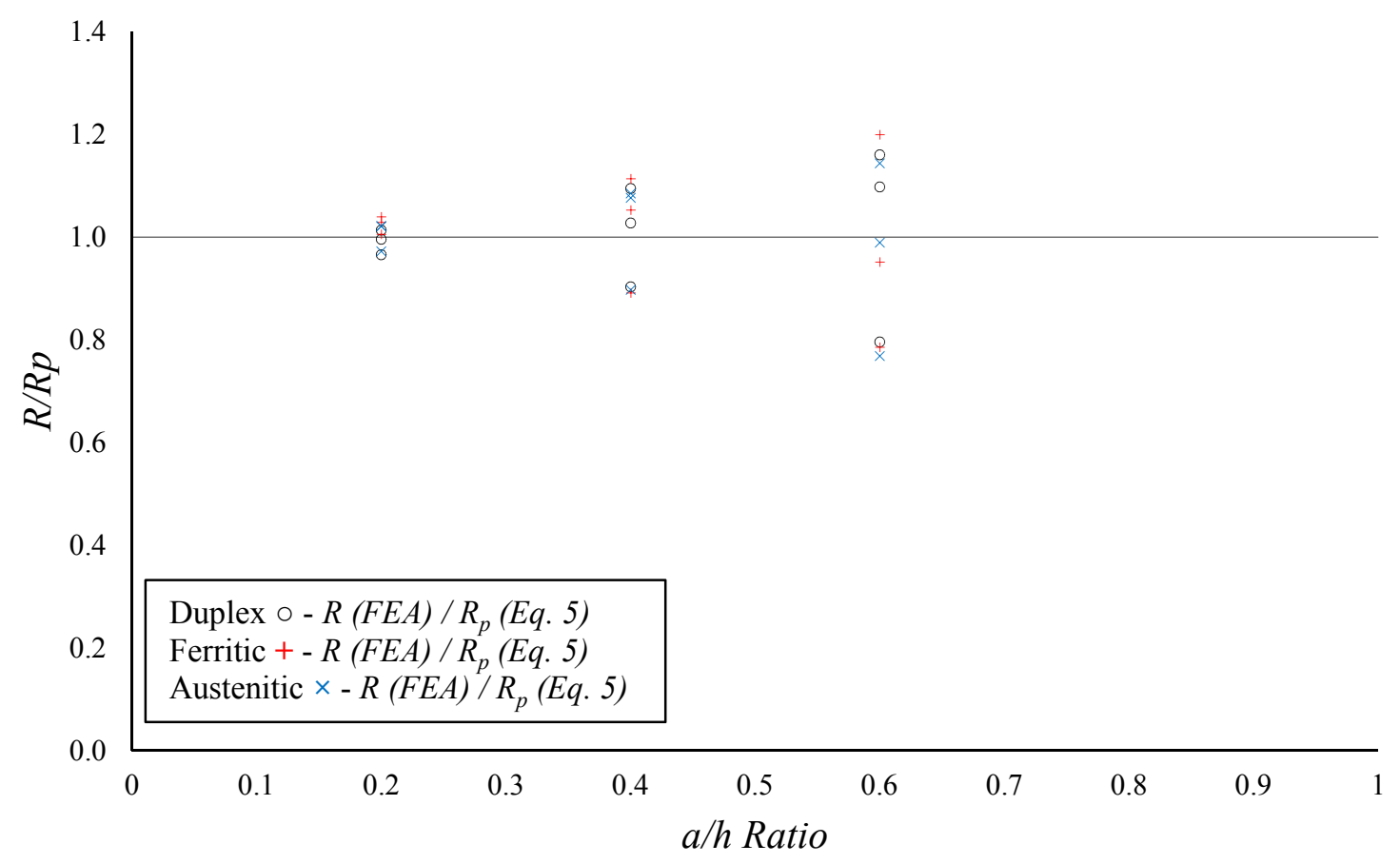

(a) Centered circular web opening

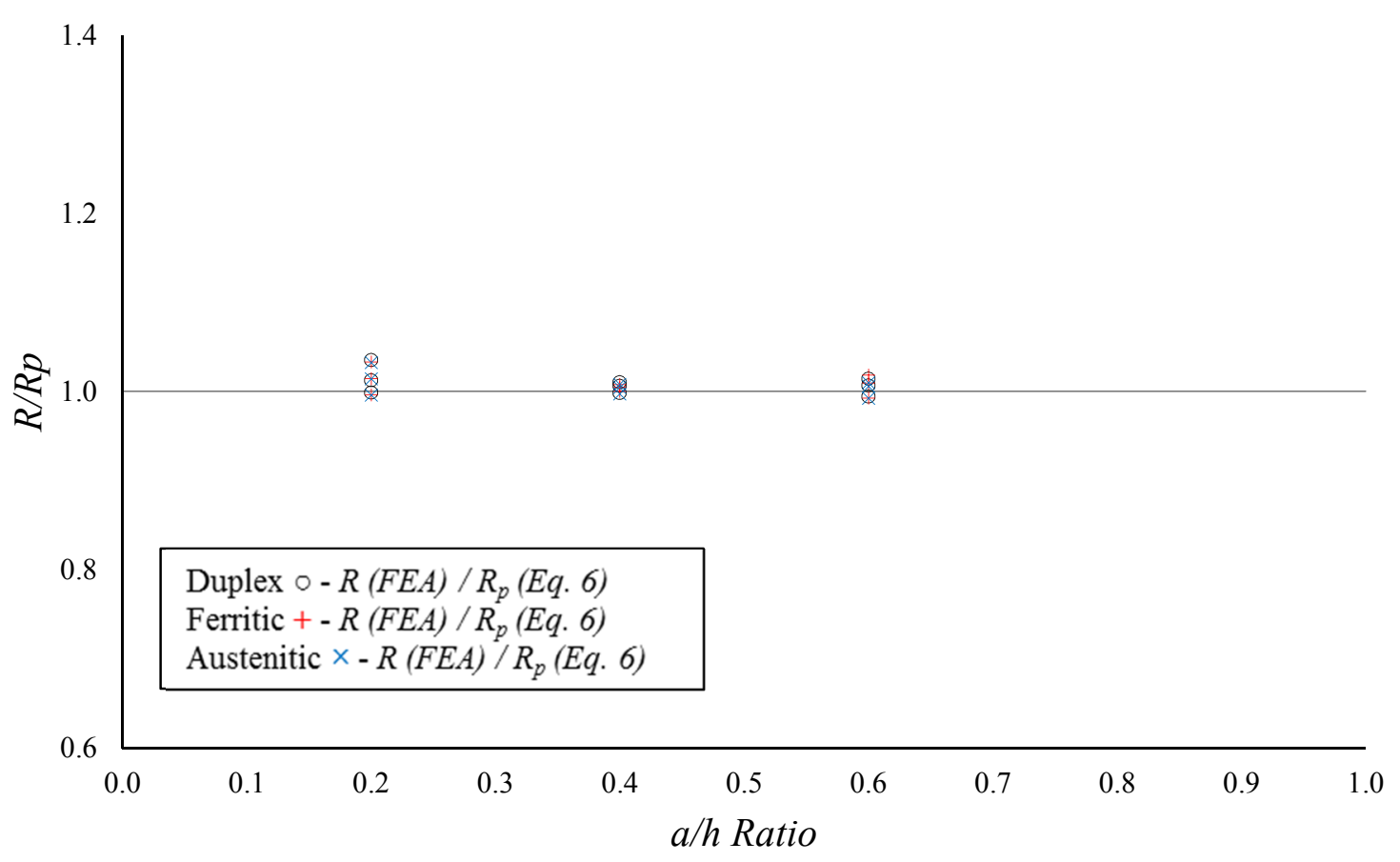

(b) Offset circular web opening

Figure 8: Comparison of strength reduction factor for C142 section for the case of flange unfastened to bearing plate 